\title{
Confined Chemical Transitions for Direct Extraction of Conductive Cellulose Nanofibers with Graphitized Carbon Shell at Low Temperature and Pressure
}

Duan-Chao Wang ${ }^{1}$, Hou-Yong $\mathrm{Yu}^{1}{ }^{1}$ *, Dongming $\mathrm{Qi}^{1}$, Yuhang $\mathrm{Wu}^{1}$, Lumin $\mathrm{Chen}^{1}$,

Ziheng $\mathrm{Li}^{1}$

${ }^{1}$ National Engineering Lab for Textile Fiber Materials \& Processing Technology, College of Textile Science and Engineering, Zhejiang Sci-Tech University, Hangzhou 310018, China

E-mail:phdyu@zstu.edu.cn 


\section{Experimental}

Materials. Ginger fiber was given from the laboratory of Jiangnan University, wheat straw, bamboo pulp, aspen wood chips, and flax fiber were homemade in the laboratory; sulfuric acid $\left(\mathrm{H}_{2} \mathrm{SO}_{4}, 98 \%\right)$, lignin (dealkalized) were purchased from Shanghai Aladdin Biochemical Technology Co., Ltd.

Table S1. Chemical composition of raw biomass materials.

\begin{tabular}{ccccc}
\hline $\begin{array}{c}\text { Raw biomass } \\
\text { material }\end{array}$ & $\begin{array}{c}\text { Cellulose } \\
(\%)\end{array}$ & $\begin{array}{c}\text { Hemicellulose } \\
(\%)\end{array}$ & $\begin{array}{c}\text { Lignin } \\
(\%)\end{array}$ & Extractives (\%) \\
\hline Ginger fiber $^{\text {a }}$ & $63-78$ & $12-16$ & $2-3$ & $4-9$ \\
Wheat straw $^{\mathrm{b}, 1}$ & $43-85$ & $6-34$ & $9-22$ & $6-8$ \\
Bamboo pulp $^{\mathrm{c}}$ & $68-75$ & $10-14$ & $2-5$ & $7-10$ \\
$\begin{array}{c}\text { Aspen wood } \\
\text { chips }\end{array}$ & $38-51$ & $15-34$ & $21-31$ & $2-4$ \\
$\begin{array}{c}\text { Flax fiber } \\
{ }^{3}\end{array}$ & $60-81$ & $14-21$ & $2-3$ & $3-7$ \\
\hline
\end{tabular}

- Omitted the number of decimal places.

a Self-test; this ginger fiber is a thick silk drawn from the dried ginger, not original fresh ginger.

${ }^{\mathrm{b}}$ Contains mechanical processed data.

${ }^{c}$ Self-test; this is bleached bamboo pulp paperboard.

Extraction of cellulose nanofibers (CNF) and confined carbonization. Firstly, $1 \mathrm{~g}$ of ginger fiber was placed into a three-neck flask and $80 \mathrm{~mL}$ of $64 \mathrm{wt} \% \mathrm{H}_{2} \mathrm{SO}_{4}$ was slowly added, taking care that the temperature of the added liquid should not be higher 
than $25^{\circ} \mathrm{C}$, completely sealed using a rubber stopper and sealant, and then passed high flux nitrogen for $30 \mathrm{~min}$. After that, the $\mathrm{CNF}$ was hydrolyzed at $40{ }^{\circ} \mathrm{C}$ for $30 \mathrm{~min}$ with stirring, sealed throughout and protected by high flux nitrogen. The second step is the confined (self-limiting) carbonization of the nanosurface of CNF: the pre-reaction solution was directly heated to $90^{\circ} \mathrm{C}$ with rapid stirring and divided into two groups for $1 \mathrm{~h}$ and $4 \mathrm{~h}$, during which the surface of CNF underwent confined dehydration and carbonization (DC) reactions, and the whole process was sealed and protected by high flux nitrogen. At the end of the reaction, the suspension was rapidly cooled to room temperature in a cold water bath, dialyzed to neutral. The samples were named DCCNFene- 1 and DC-CNFene- 4 according to the reaction time, and these two samples were used for experimental reaction mechanism and confined transform structure analysis.

Extraction of pure CNF. $1 \mathrm{~g}$ of ginger fiber was placed into a three-neck flask and 80 $\mathrm{mL}$ of $64 \mathrm{wt} \% \mathrm{H}_{2} \mathrm{SO}_{4}$ was slowly added, taking care that the temperature of the added liquid should not be higher than $25^{\circ} \mathrm{C}$, sealing and nitrogen protection. After that, the $\mathrm{CNF}$ was hydrolyzed at $40{ }^{\circ} \mathrm{C}$ for 30 min with stirring. After cooling to room temperature, pure CNF was obtained by dialyzing to neutral.

Extraction of DC CNF without oxygen isolation (DC-O-CNF). Except for no need for sealing and nitrogen protection, the experimental procedures of these two sets of samples were the same as CNFene. According to the reaction time of one hour or four hours, because they will carry more oxygen-containing groups, the samples were named $\mathrm{DC}-\mathrm{O}-\mathrm{CNF}-1$ and $\mathrm{DC}-\mathrm{O}-\mathrm{CNF}-4$, respectively. 
Extraction of CNFene with low biomass weight ratio. This group ginger fibers were treated with the same reaction method as $\mathrm{DC}-\mathrm{CNFene}-4$, but the ginger fibers were only used $0.2 \mathrm{~g} / 80 \mathrm{~mL} 64 \% \mathrm{wt} \mathrm{H}_{2} \mathrm{SO}_{4}$.

Generalized experiments of CNFene preparation by Confined DC. Wheat straw, bamboo pulp, aspen wood chips, and flax fibers were treated with the same reaction method as ginger fiber, but only $4 \mathrm{~h}$ reaction set was performed, named $\mathrm{DC}-\mathrm{CNFene}-$ Straw, DC-CNFene-Bamboo, DC-CNFene-Wood, and DC-CNFene-Flax, respectively.

CNFene morphology characterization. The intrinsically conducting CNFene morphology was characterized by field emission scanning electron microscopy (FESEM), high resolution transmission electron microscopy (HRTEM), and atomic force microscope (AFM): the overall morphology was observed by FE-SEM at an accelerating voltage of $1.0 \mathrm{kV}$, and about $0.01 \mathrm{wt} \%$ of CNFene fibers were dispersed in water and the suspension was sonicated for $15 \mathrm{~min}$, then deposited on silicon wafers and dried in an oven at $60{ }^{\circ} \mathrm{C}$ for direct observation without gold plating. Welldispersed CNFene fibers were observed by HRTEM, and deposits of aqueous suspensions of about $0.002-0.01 \mathrm{wt} \%$ of the sample were deposited on ultrathin carbon grids for observation, high-resolution images were taken, selected area electron diffraction (SAED) and X-ray energy spectroscopy (EDS) analysis was performed. To study the morphology of the CNFene Derived graphene film, the AFM (Bruker Dimension Icon and Park XEI-100) with nanoscopic software were used. The scanning was operated in taping mode and the height image was collected. For the sample 
preparation, a drop of DC-CNFene-4 suspension after 10 min of sonication was cast on $1 \times 1 \mathrm{~cm}$ silicon wafer and dried in the oven.

Raman spectroscopy. Raman spectra of CNFene were acquired using a $532 \mathrm{~nm}$ laser in the range of $1000-2000 \mathrm{~cm}^{-1}$ with a step size of $2 \mathrm{~cm}^{-1}$. The degree of graphitization and the percentage of defects in the highly graphitized carbon layer were evaluated by comparing the peaks in the $\mathrm{D}$ and $\mathrm{G}$ bands.

X-ray diffraction (XRD). CNFene were pressed into slices and placed on slides to record XRD patterns in the test range of $5-80^{\circ}$ with a step size of $1 \% / \mathrm{min}$.

Ultraviolet-visible (UV-Vis) spectroscopy. The optical properties of CNFene were characterized by using a UV spectrophotometer in the wavelength range of 200 to 600 $\mathrm{nm}$. Please note that tiny fluctuations at $\sim 340 \mathrm{~nm}$ during UV instrument testing are generally not characteristic peak of these samples, but rather a fluctuations of the instrument use a tungsten light source (WI) in the visible region and switched to a xenon light source (D2) in the UV region.

Thermogravimetric analysis (TG). Samples (3-8 mg) were heated from 30 to $800{ }^{\circ} \mathrm{C}$ at $20^{\circ} \mathrm{C} / \mathrm{min}$ while protected using a dynamic nitrogen atmosphere. The main thermal parameters such as initial decomposition temperature $\left(\mathrm{T}_{0}\right)$, maximum decomposition temperature $\left(\mathrm{T}_{\max }\right)$ and residual mass $\left(\mathrm{M}_{\mathrm{r}}\right)$ were recorded from the TGA curves.

TG-FTIR: The decomposition gases generated during the thermal degradation of TG were transferred to a high temperature infrared gas cell using nitrogen gas through a high temperature gas conductor. The spectrometer was used in absorption mode with a wavelength range of 4000 to $400 \mathrm{~cm}^{-1}$. For each spectrum, $8 \mathrm{~cm}^{-1}$ resolution and 8 
spectral recordings were used. Separate background spectra were collected and subtracted from the original spectra of each sample. Finally each sample was synthesized from these 230 FTIR spectra of decomposed gases into a TG-FTIR (temperature-wavelength) two-dimensional spectrum and a three-dimensional spectrum, from which the composition of the substances decomposed during thermal degradation of the samples could be analyzed and distinguished in which temperature band they were decomposed.

X-ray photoelectron spectroscopy (XPS). the XPS spectrometer was operated in constant analyzer energy (CAE) mode, using a $1 \mathrm{eV}$ step size and an $\mathrm{Al} \mathrm{K}_{\alpha} \mathrm{X}$-ray source (1486.6 eV), the sample was lightly pressed (10-7 Pa) and mounted on a double-sided tape for analysis. The $\mathrm{C} / \mathrm{O}$ ratio and $\mathrm{S}$ element content were calculated by full spectrum analysis, and split-peak fitting was performed by Avantage software to identify the chemical states and percentages of different valence states of $\mathrm{C}$ for the $\mathrm{C} 1 \mathrm{~s}$ spectrum, and the different chemical states and percentages of $\mathrm{O}$ for the $\mathrm{O} 1 \mathrm{~s}$ spectrum.

Electrochemical testing of intrinsically conducting CNFene. Cyclic voltammetry (CV) and impedance (EIS) studies were performed using a CHI660E electrochemical analyzer: $\mathrm{CV}$ scan data were obtained in a voltage window of $0.0-1.0 \mathrm{~V}$ using $\mathrm{H}_{2} \mathrm{SO}_{4}$ as the electrolyte and a scan rate of $10-400 \mathrm{mV} / \mathrm{s}$. The total capacitance was calculated using half of the integrated area of the CV curve as follows.

$$
C_{\mathrm{m}}=\left(\int I / d V\right) /(2 v m \Delta V)
$$

where $v(\mathrm{~V} / \mathrm{s})$ is the scan rate, where $I(\mathrm{~A})$ is the discharge current, $\Delta t(\mathrm{~s})$ is the discharge time, $m(\mathrm{~g})$ is the total mass of the electrode, and $\Delta V(\mathrm{~V})$ is the operating 
discharge voltage window.

The EIS measurements were performed in the frequency range of $0.01 \mathrm{~Hz}-300 \mathrm{kHz}$. $\mathbf{N}_{2}$ absorption-desorption analysis. The absorption-desorption isotherms were collected by a BEISHIDE PS1 Surface Area and Porosimetry Analyzer. All the samples were kept at $100{ }^{\circ} \mathrm{C}$ for $2 \mathrm{~h}$ for dewatering. $\mathrm{N}_{2}$ isotherms were measured at $77.3 \mathrm{~K}$. 
a

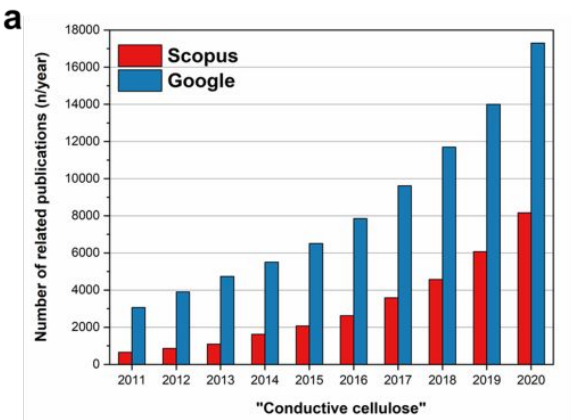

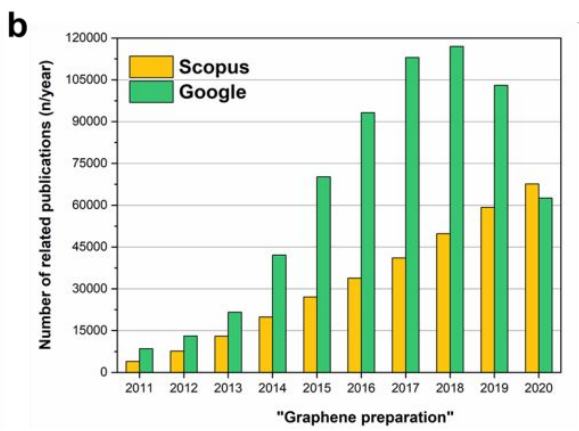

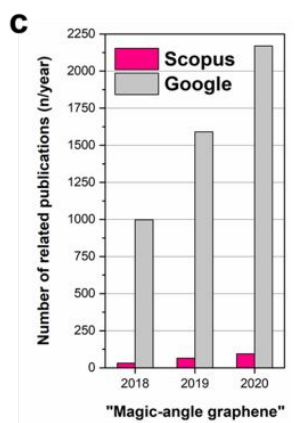

Figure S1. Research interest in conductive cellulose and graphene reflected in the number of academic papers year by year.
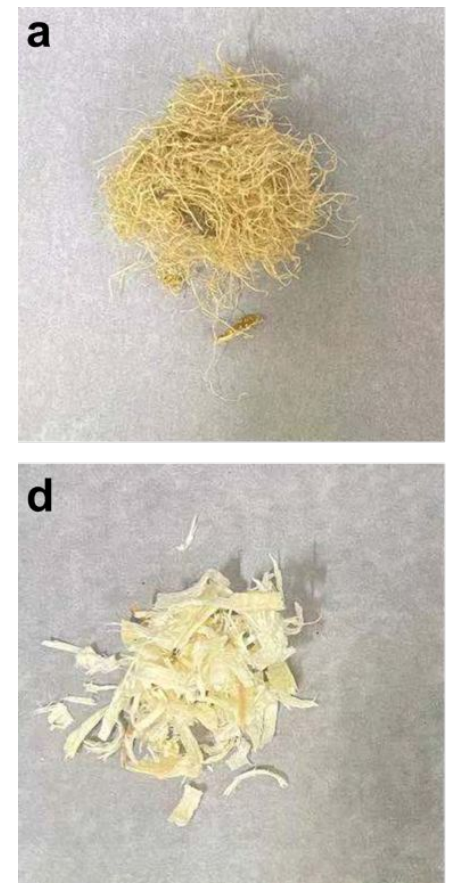
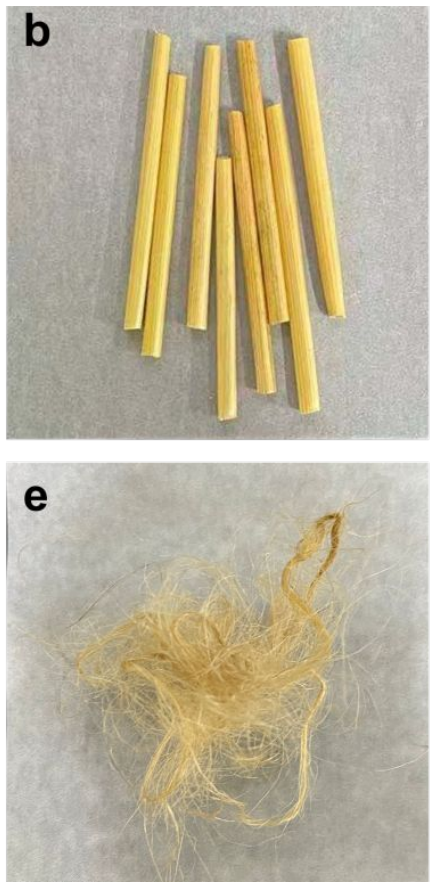
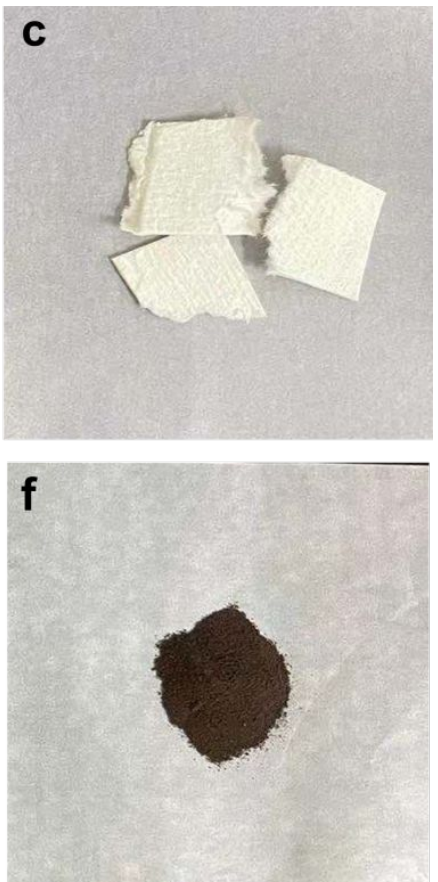

Figure S2. Photos of (a) ginger fibers, (b) wheat straw, (c) bamboo pulp board, (d) aspen wood chips, (d) flax fiber, and (e) lignin. Photos size $10 \times 10 \mathrm{~cm}$. 

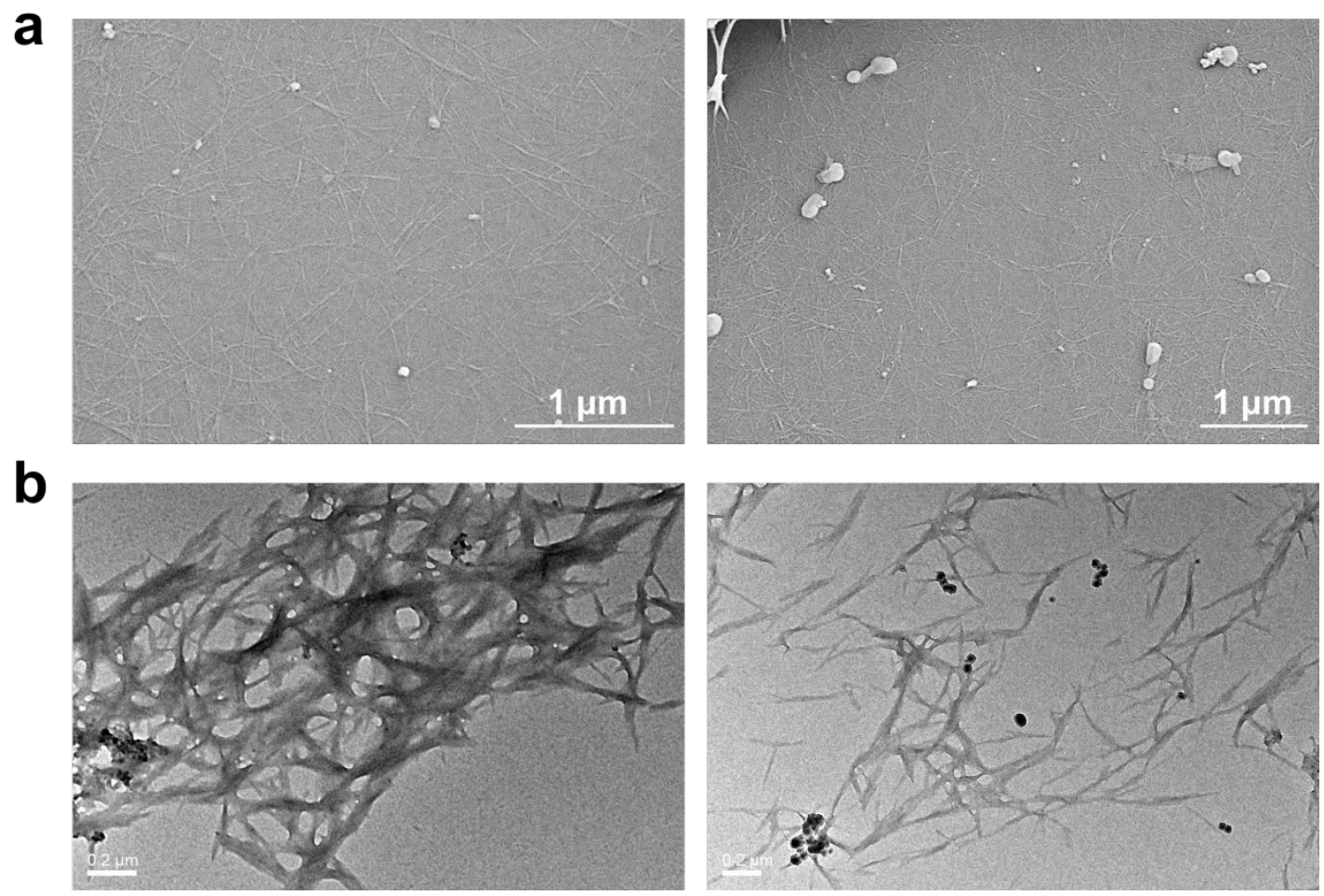

Figure S3. (a) FE-SEM images and (b) TEM images of original CNF extracted from ginger fiber by $\mathrm{H}_{2} \mathrm{SO}_{4}$ hydrolysis.

According to statistical data, the length of CNF was 1385-2686 nm and the diameter was $36-108 \mathrm{~nm}$. 
1. Acid hydrolysis, $40^{\circ} \mathrm{C}, \mathrm{N}_{2}$

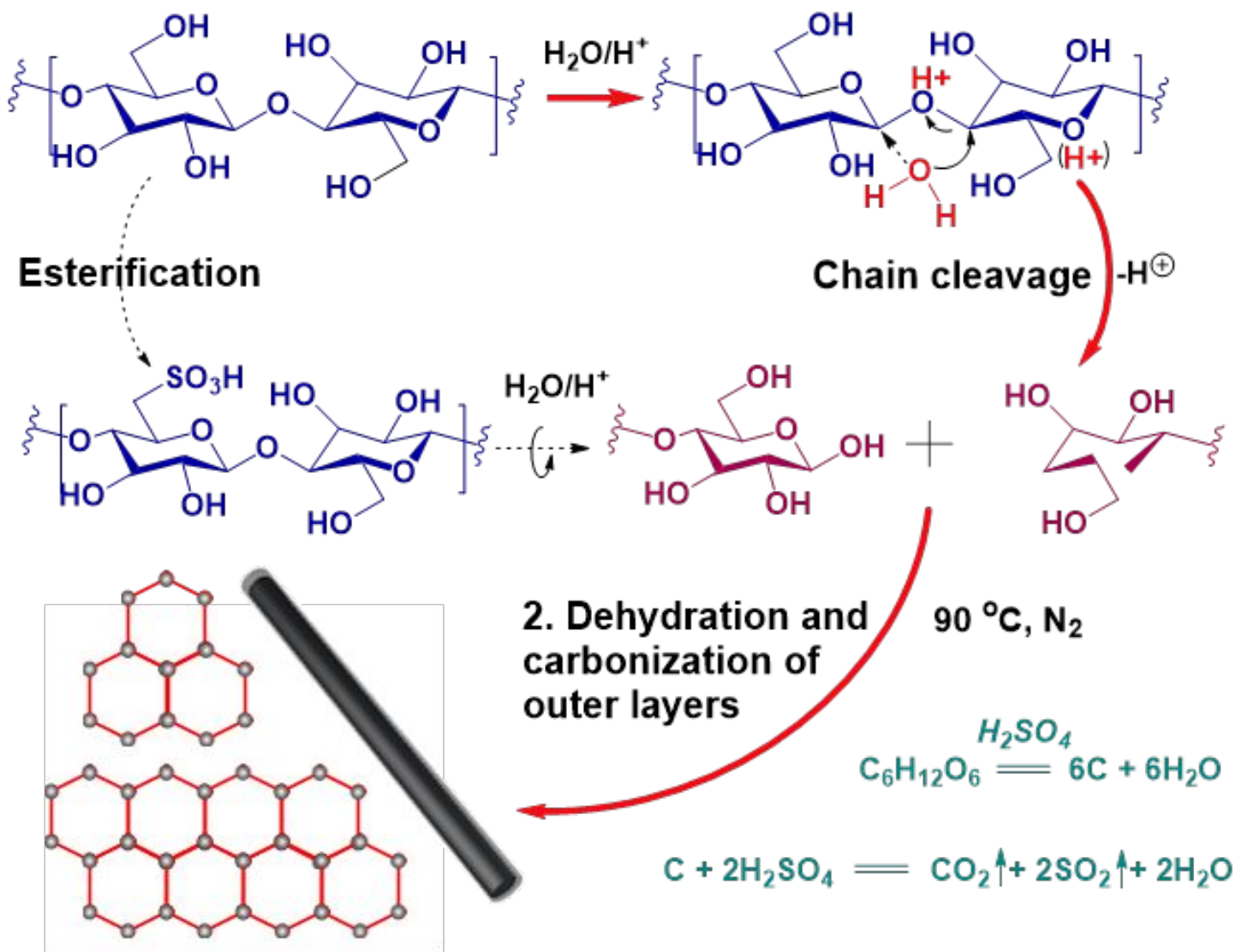

Figure S4. Schematic diagram of confined DC method to fabricate CNFene and derived graphene. 


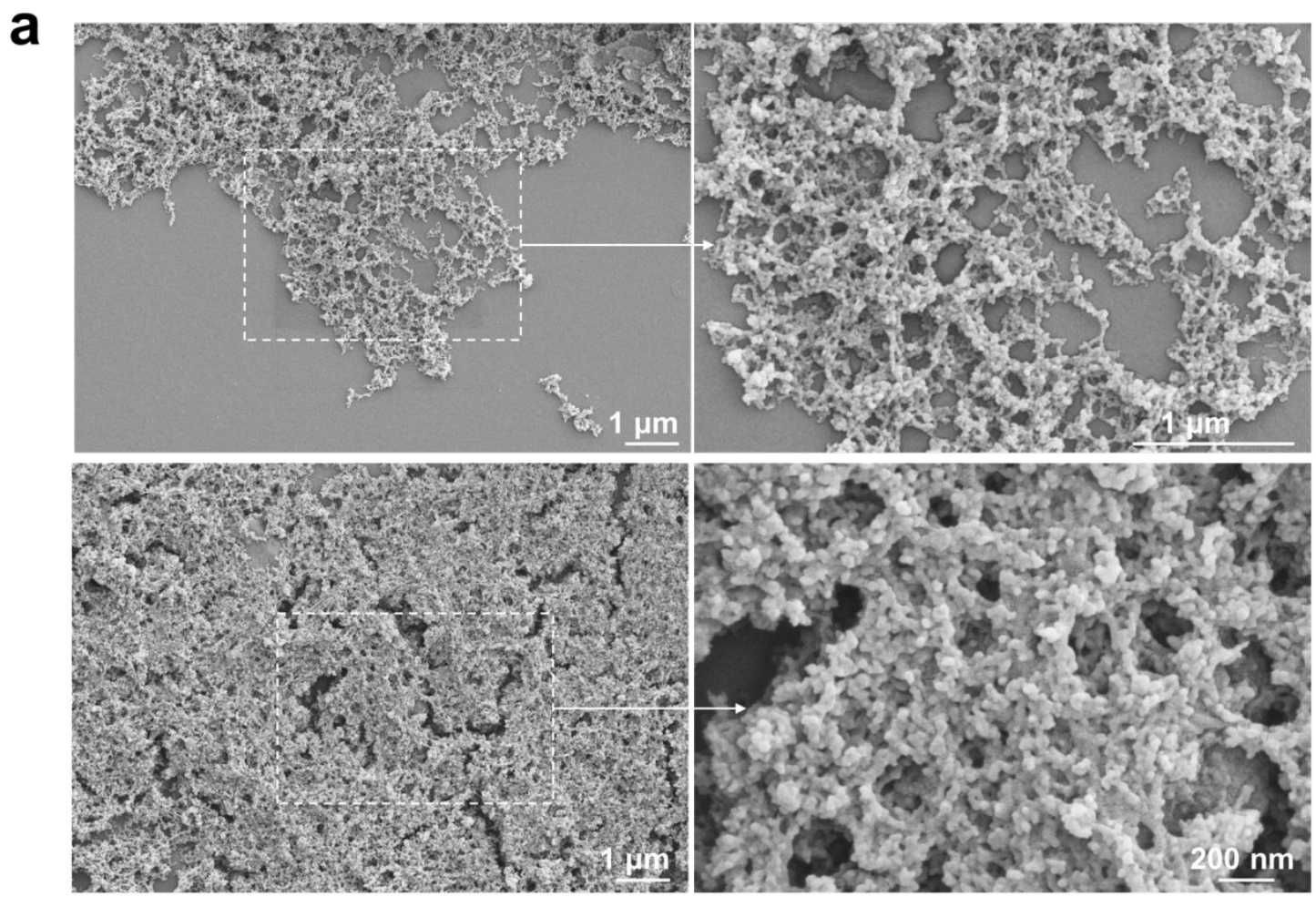

b
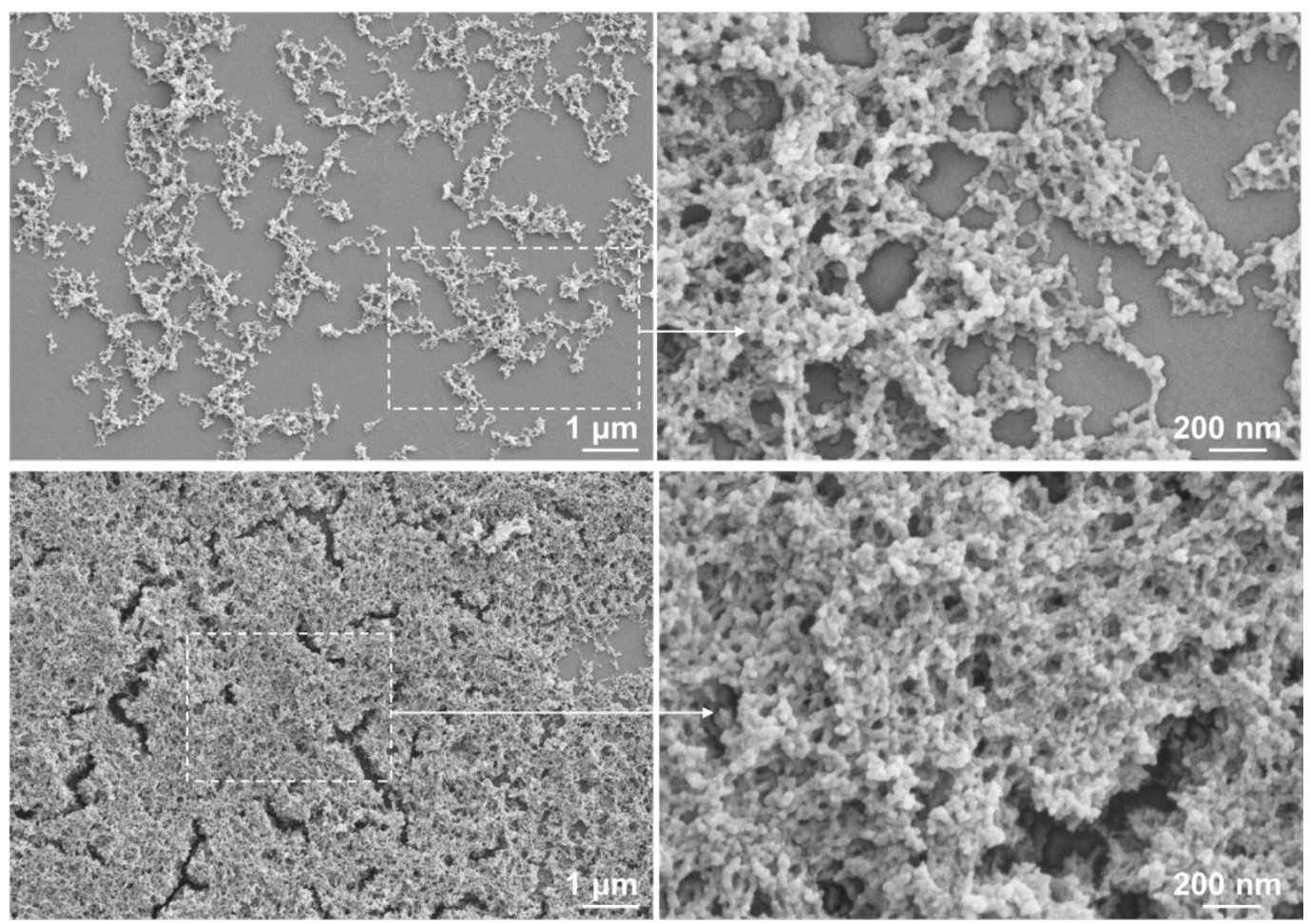

Figure S5. FE-SEM images of (a) DC-CNFene-1 and (b) DC-CNFene-4. 
a
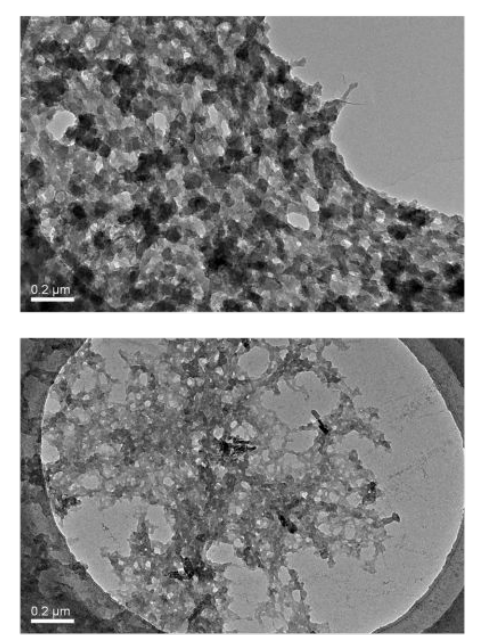

b
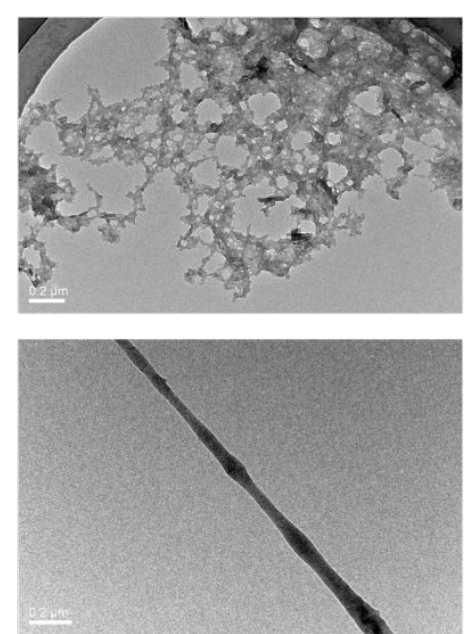

Figure S6. HRTEM images of (a) DC-CNFene-1 and (b) DC-CNFene-4.
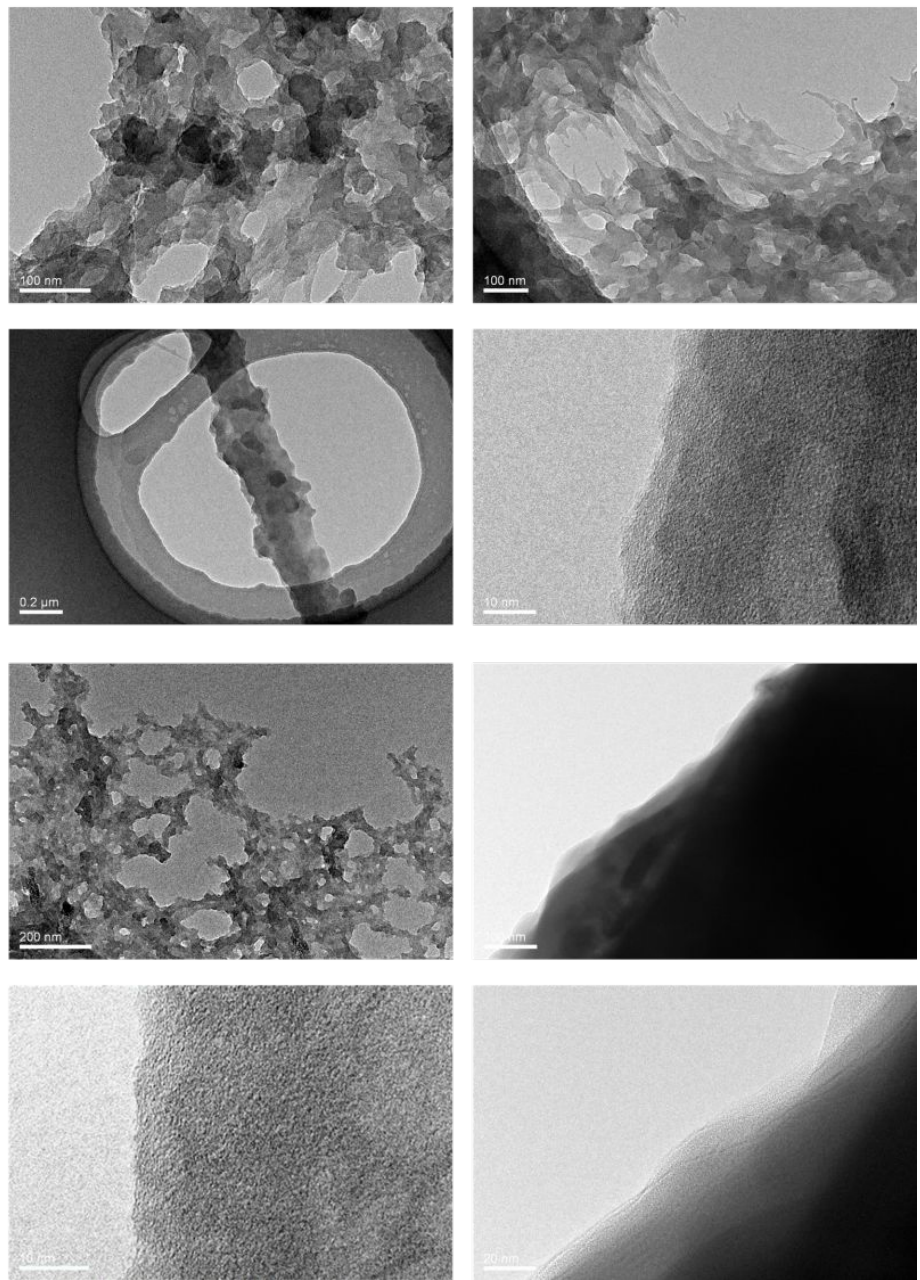
a

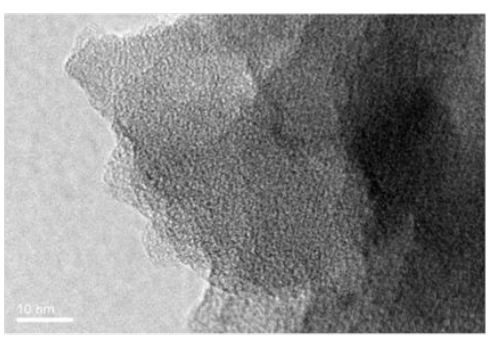

C

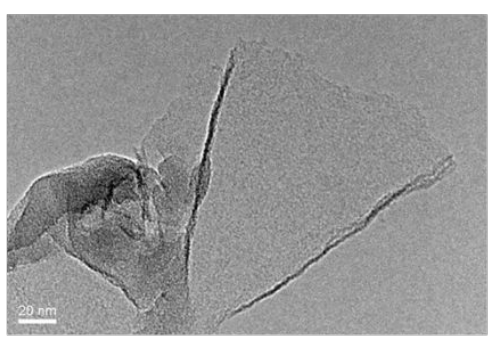

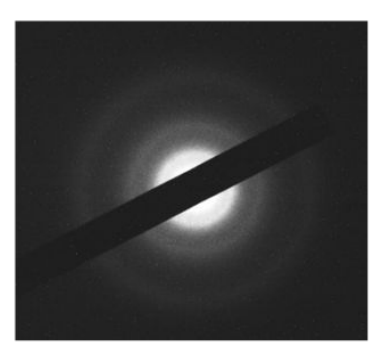

b
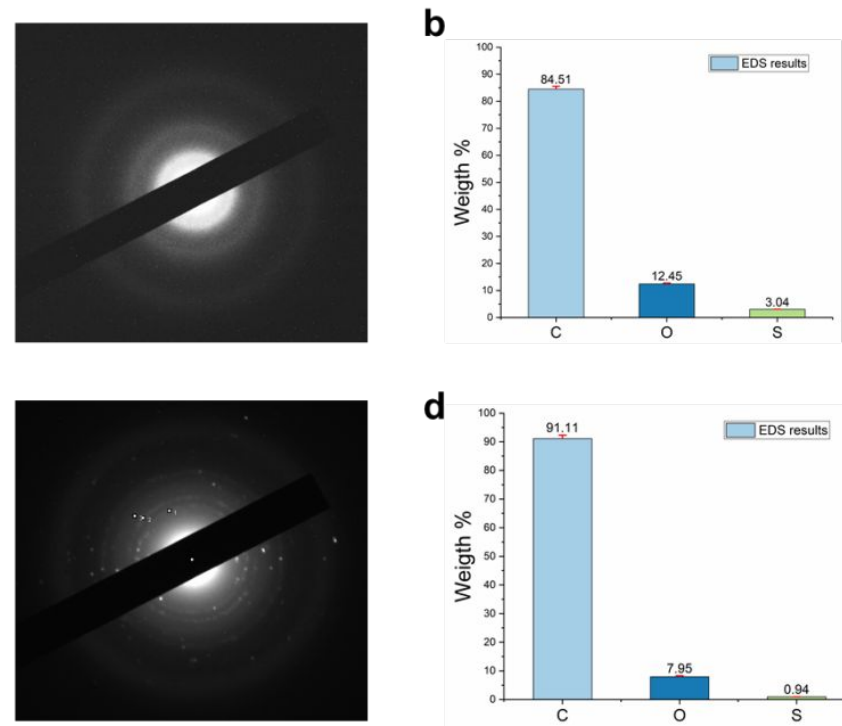

d

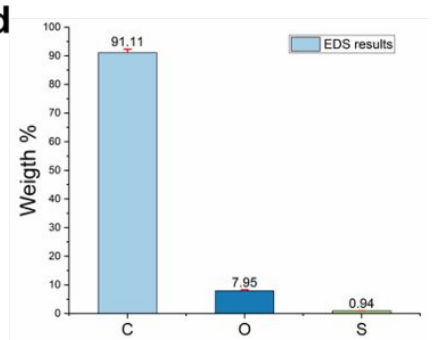

Figure S7. (a) TEM images and SAED of DC-CNFene-1 derived graphene and (b) its elements ratio of $\mathrm{C}, \mathrm{O}$, and $\mathrm{S}$ from EDS results. (c) TEM images and SAED of DCCNFene- 4 derived graphene and (b) its elements ratio of $\mathrm{C}, \mathrm{O}$, and $\mathrm{S}$ from EDS results.

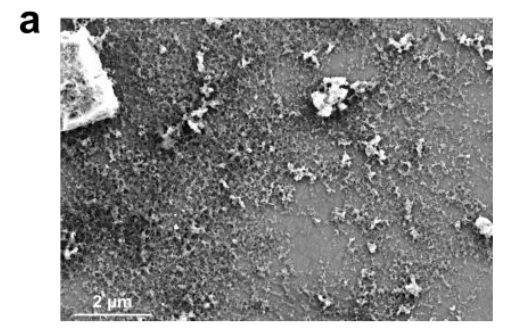

b
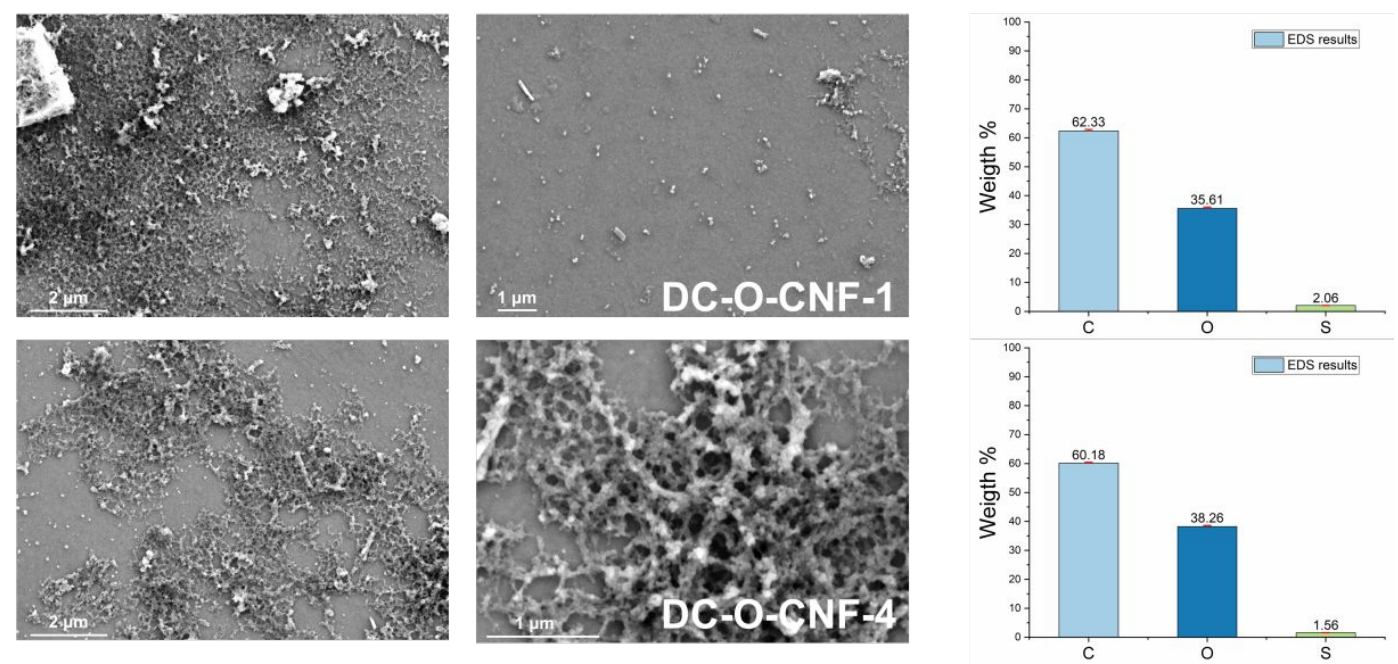

Figure S8. Samples obtained when the preparation process was not isolated from oxygen. (a) FE-SEM images of $\mathrm{DC}-\mathrm{O}-\mathrm{CNF}-1$ and its elements ratio from EDS results. (b) FE-SEM images of DC-O-CNF-4 and its elements ratio from EDS results. 

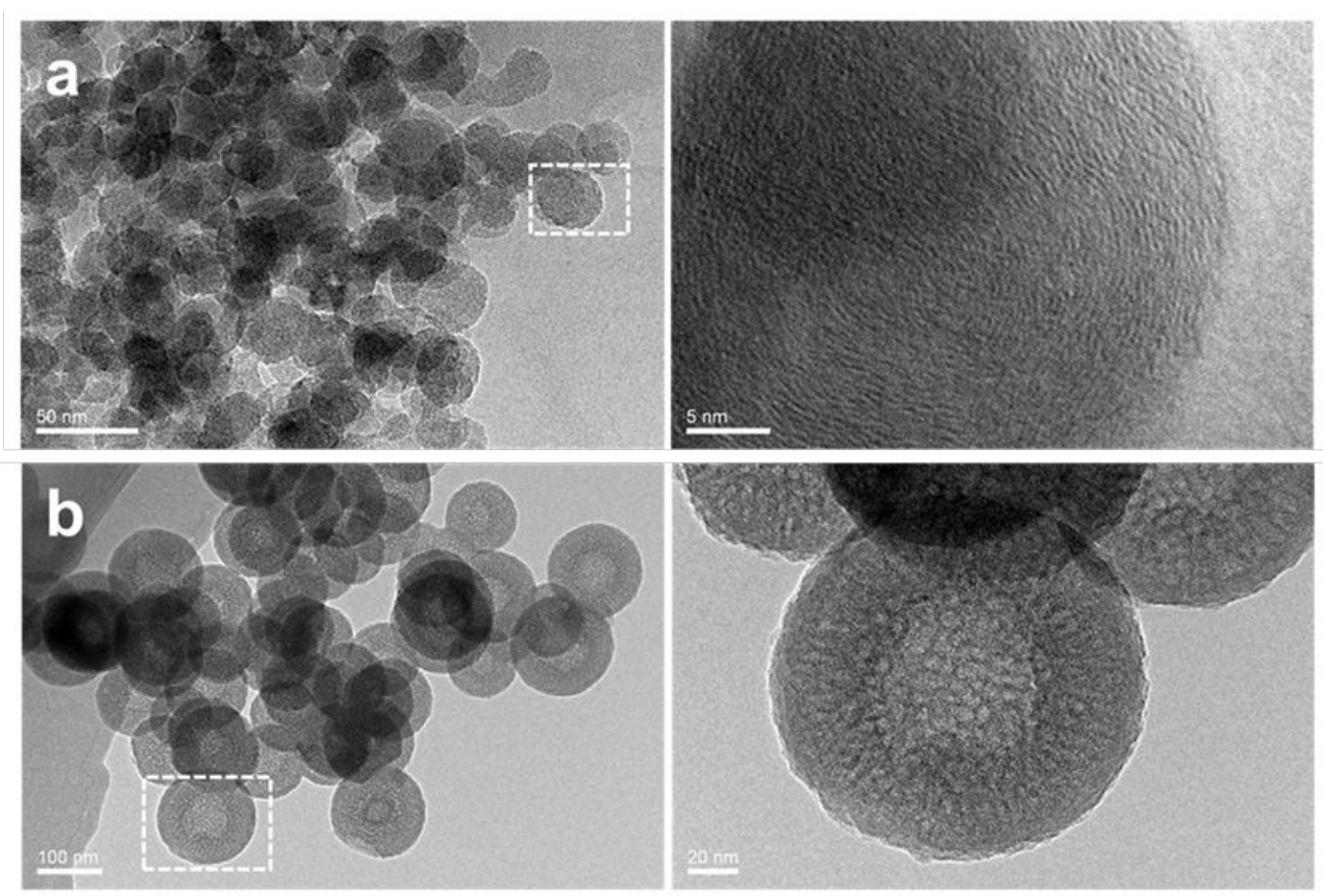

Figure S9. TEM images of (a) of DC-O-CNF-1 and (b) DC-O-CNF-4 nanospheres.

For the Raman spectrum, the total scattered light intensity $\left(I_{s}\right)$ integrated from the surface to the depth $\mathrm{d}$ is expressed as ${ }^{4-5}$ :

$$
I_{s}=I_{0} D \int_{0}^{d} e^{-2 \alpha x} d x=\frac{I_{0} D}{2 \alpha}\left(1-e^{-2 \alpha d}\right)
$$

The light intensity $\left(I_{d}\right)$ from depth $(d)$ to infinity is in accordance with the following formula:

$$
I_{d}=I_{0} D \int_{d}^{\infty} e^{-2 \alpha x} d x=\frac{I_{0} D}{2 \alpha} e^{-2 \alpha d}
$$

Among them, $I_{0}, D$, and $\alpha$ are the intensity of incident light, the Raman scattering cross section and the light absorption coefficient, respectively. Assuming that the laser penetration depth $d_{p}$ is the same as the depth $\mathrm{d}$ and satisfies the relationship of $I_{d} /\left(I_{s}+I_{d}\right)$ $=0.1$, the penetration depth $\mathrm{dp}$ can be given by the following formula ${ }^{6-7}$ :

$$
d_{p}=\frac{-\ln 0.1}{2 \alpha}=\frac{2.3}{2 \alpha}
$$


Based on this calculation, the $532 \mathrm{~nm}$ laser characterized by Raman has a penetration depth of $935 \mathrm{~nm}$ for silicon and a detection depth of $21 \mathrm{~nm}$ for germanium ${ }^{8}$, so it is easy to penetrate a highly graphitized carbon layer of about $20 \mathrm{~nm}$. On the optical path, the laser will excite the Raman signal of the photon-interacting molecules, so the highly graphitized carbon layer will be excited by the Raman signal, but if the highly graphitized carbon layer is too thin, the total amount of excited Raman signal will be too small. It will be interfered by noise or other Raman signals, that is, the signal of the internal cellulose structure may produce corresponding interference. In addition, the detection depth of XRD can reach tens of microns ${ }^{9}$, the absorbance mode of UVVis is also completely penetrating to the dilute suspension of CNFene, and TG directly tests all substances regardless of the sample size. Therefore, the results of several characterizations are expected to have the characteristics of graphene and cellulose at the same time.

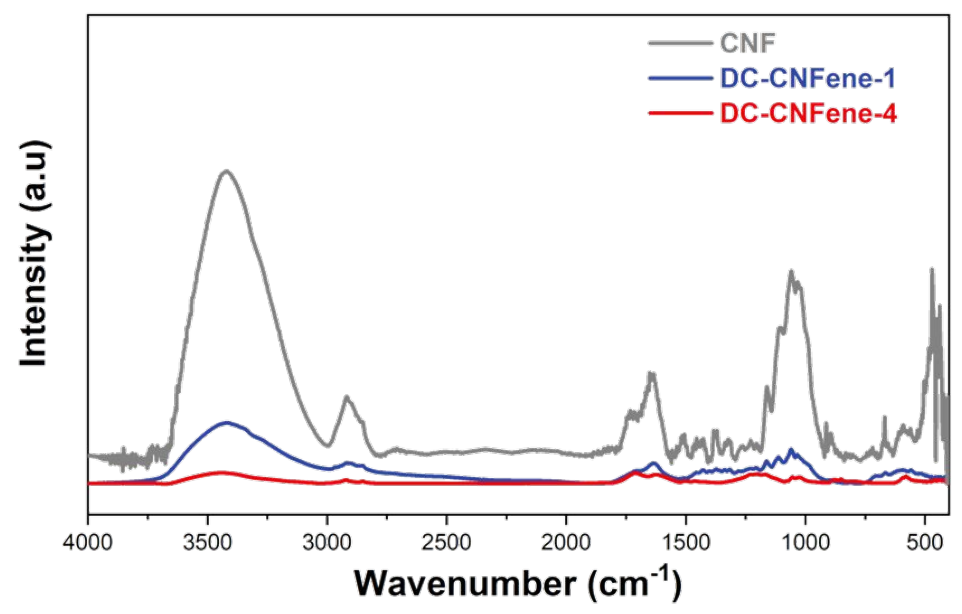

Figure S10. FTIR spectra of (a) CNF, (b) DC-CNFene-1, and (c) DC-CNFene-4. All spectra have been normalized. 
a

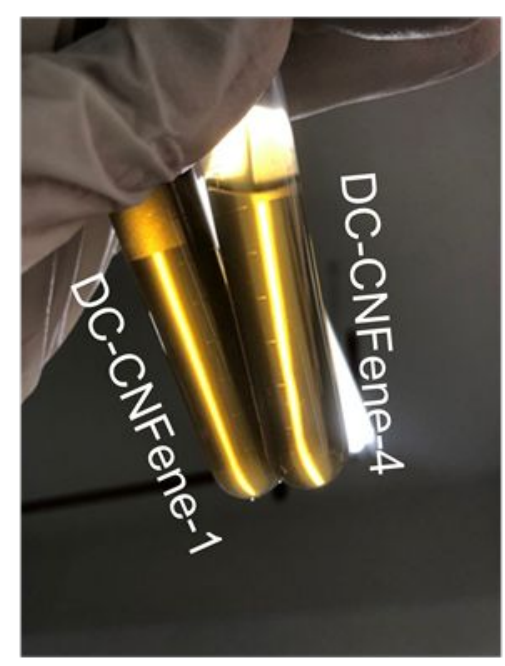

b

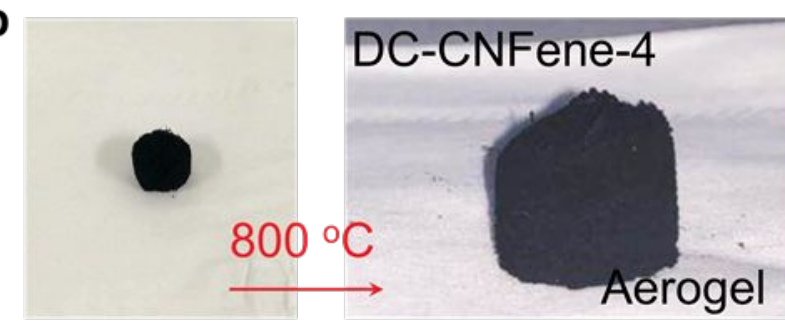

C

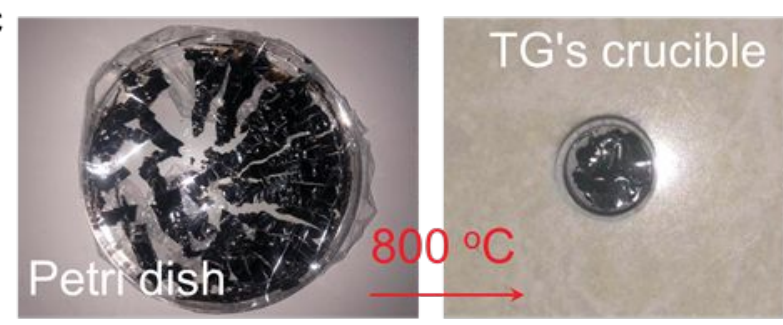

Figure S11. (a) A photo of $0.1 \mathrm{wt} \%$ suspension of DC-CNFene-1 and DC-CNFene- 4 .

(b) Photos of freeze-dried DC-CNFene-4 aerogel calcined at $800^{\circ} \mathrm{C}$. (c) Photos of oven-dried DC-CNFene- 4 aerogel calcined at $800{ }^{\circ} \mathrm{C}$.
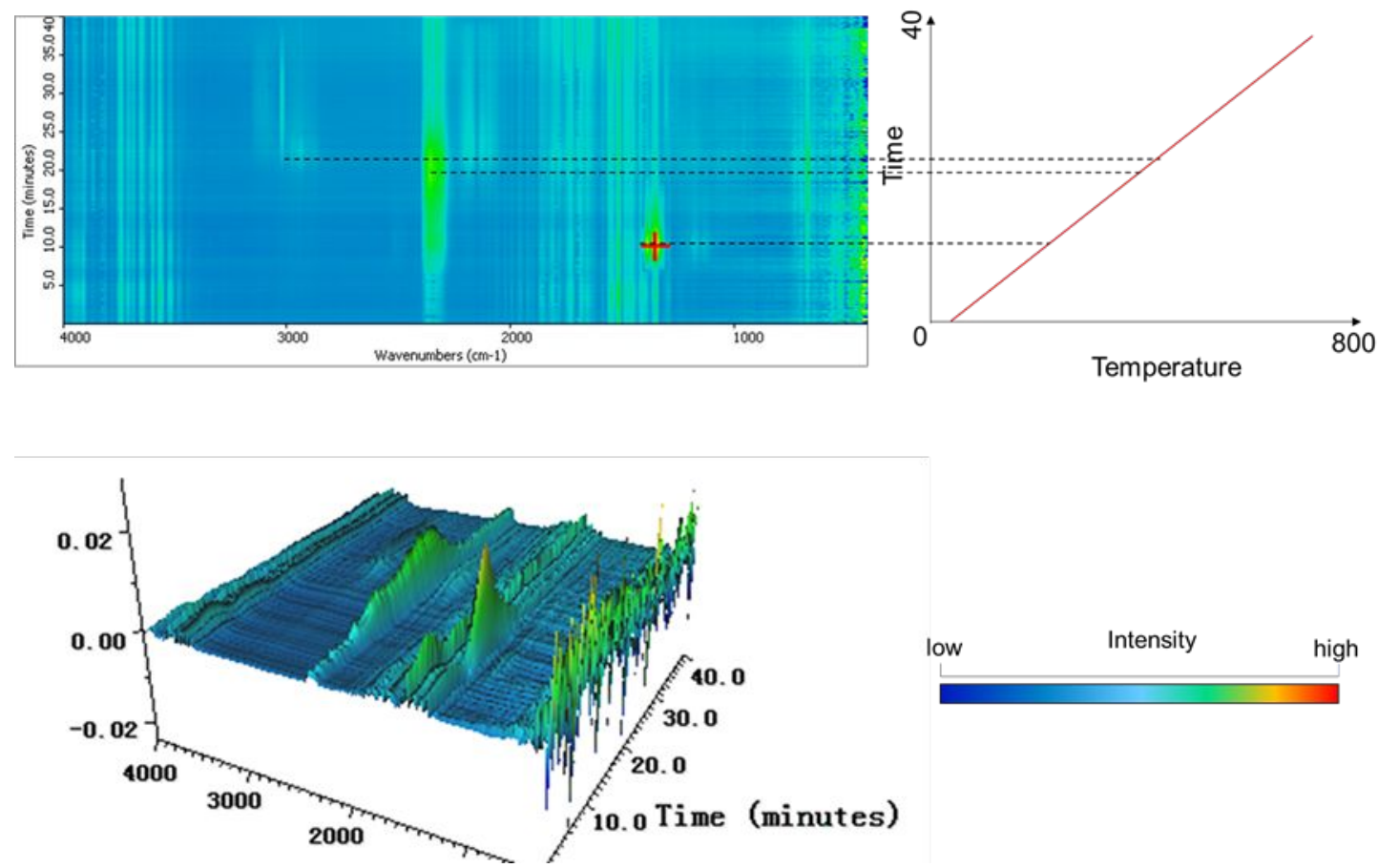

Figure S12. 2D and 3D TG-FTIR spectra of DC-CNFene-1. 
XPS is the best choice for characterizing the surface chemical structure of CNFene, because its detection depth is lower than the average thickness of CNFene's highly graphitized carbon layers, which can avoid signal interference from the internal cellulose structure. The principle of the XPS instrument is to interact with the atoms on the surface of the sample through incident electrons, and release electrons and particles with the characteristics of the atomic signals on the sample surface through various physical effects of energy transfer. The thickness of the highly graphitized carbon layer of CNFene studied in this part is about $15 \mathrm{~nm}$. Whether XPS can penetrate the highly graphitized carbon layer can be evaluated by calculation. When the photoelectrons of XPS are injected along the normal direction of the sample, the relationship between the intensity of the emitted electrons and the depth is compounded by Lambert's exponential decay law ${ }^{10}$, and the depth $d$ is taken as $3 \lambda$ :

$$
I_{d}=I_{0} \exp (-d / \lambda)
$$

Among them, $I_{d}$ is the signal intensity at depth $d ; I_{0}$ is the signal intensity at depth $0 ; \lambda$ is the electron escape depth, the inelastic scattering free path of the escaped electron, and the organic matter is $4-10 \mathrm{~nm}$.

The function curve of XPS signal intensity and electron incidence depth calculated according to the above formula is shown in Figure 4a, when $d=3 \lambda, I_{d}=0.05 I_{0}$, so in the conventional XPS test, $95 \%$ of the signal comes from a depth less than $3 \lambda$, that is, within a depth of $10 \mathrm{~nm}$. 

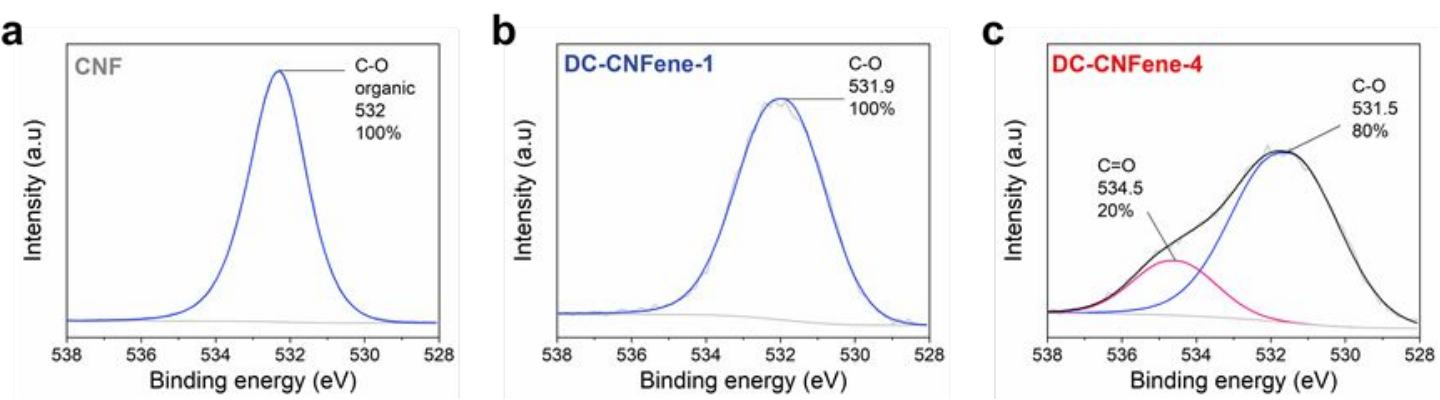

Figure S13. O 1s peaks of (a) CNF, (b) DC-CNFene-1, and (c) DC-CNFene-4.

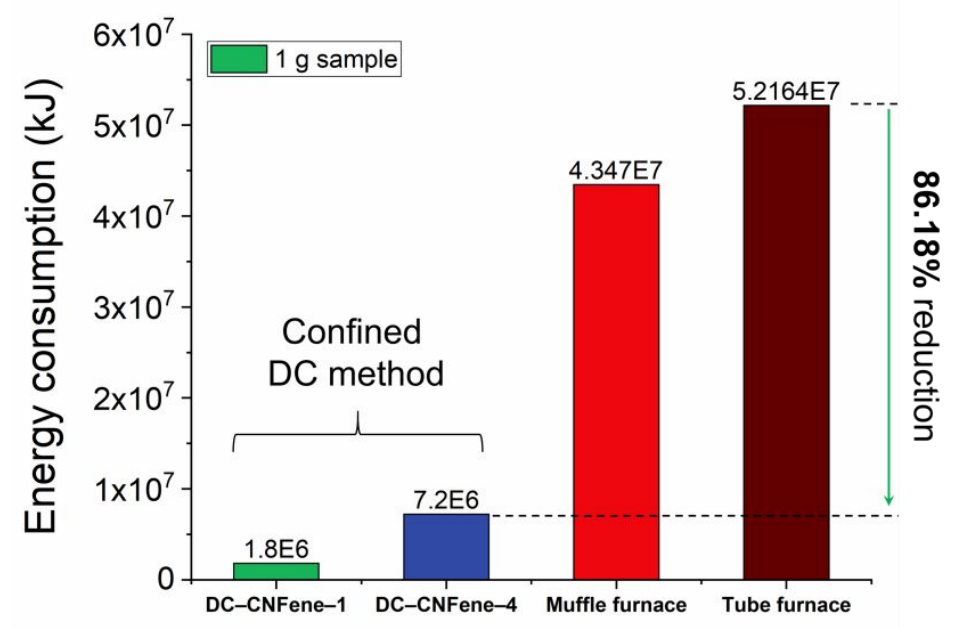

Figure S14. The energy consumption comparison between the DC method in this study and the traditional pyrolysis method.

Note: The rated power of the thermostatic water bath heater used in this laboratory is $500 \mathrm{~W}$. The rated power of the muffle furnace is $2.5 \mathrm{~kW}$, and tube furnace is $3 \mathrm{~kW}$, the work time from temperature 25 to $900{ }^{\circ} \mathrm{C}$ is generally $4.83 \mathrm{~h}$. 

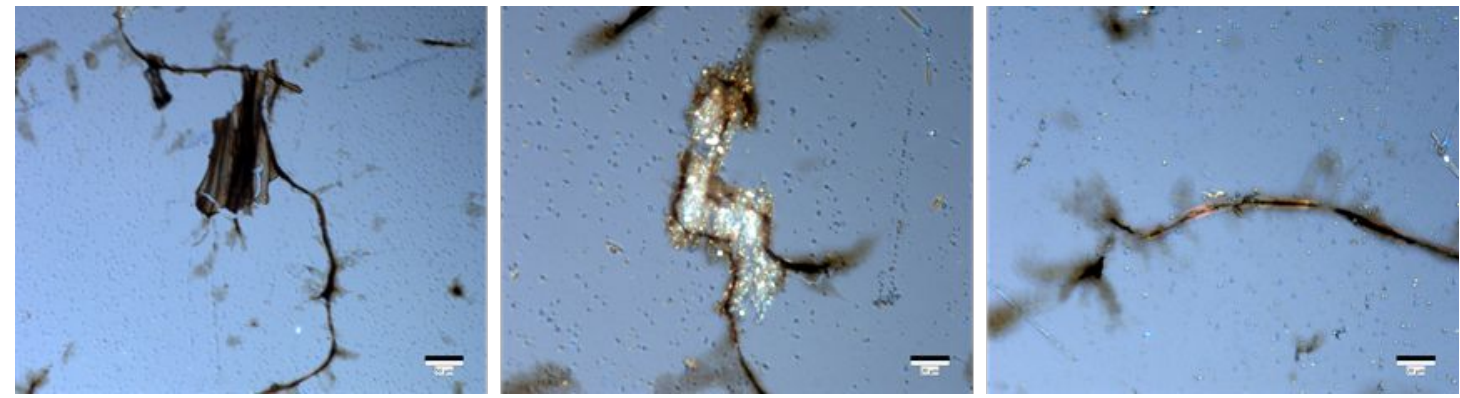

Figure S15. POM images of DC-CNFene-4 and derived graphene microcrystals or films.

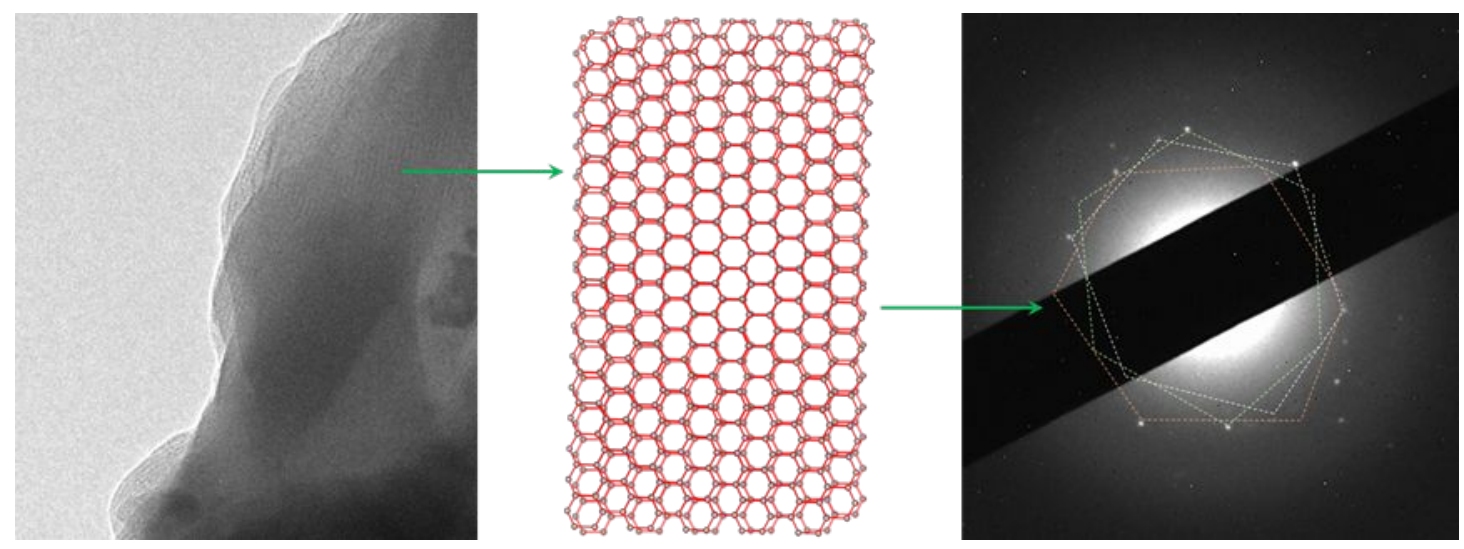

Figure S16. Moire patterns and SAED of DC-CNFene-4 derived graphene film.

a
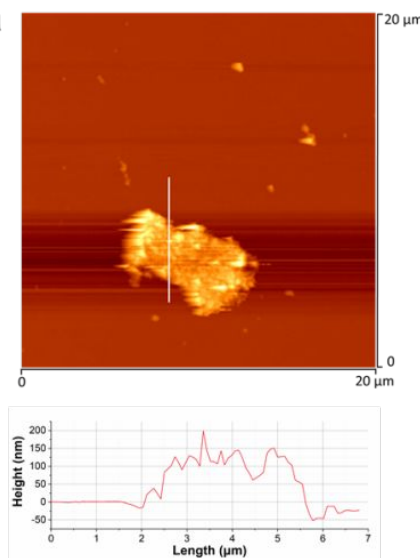

b
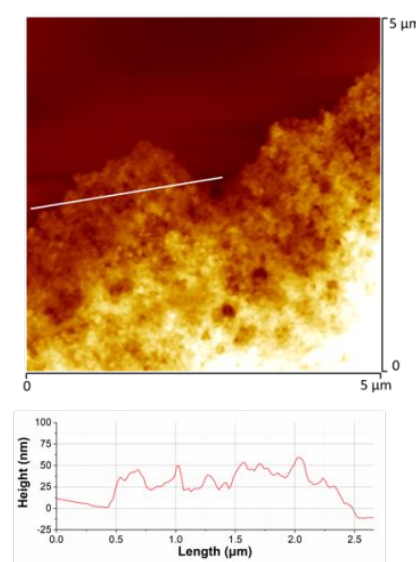

C
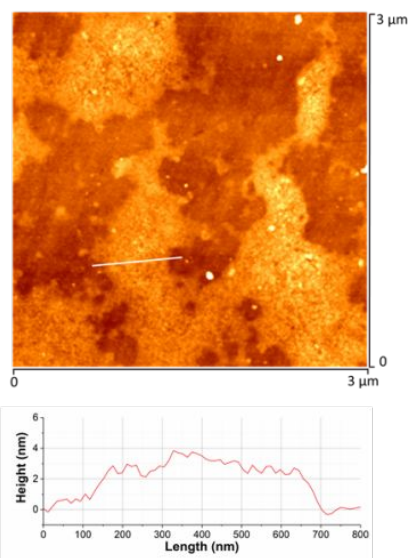

Figure S17. AFM images of DC-CNFene- 4 derived graphene films and their height distribution. (a-b) Thicker graphene films that were visible to the naked eye. (c) Invisible graphene films. 

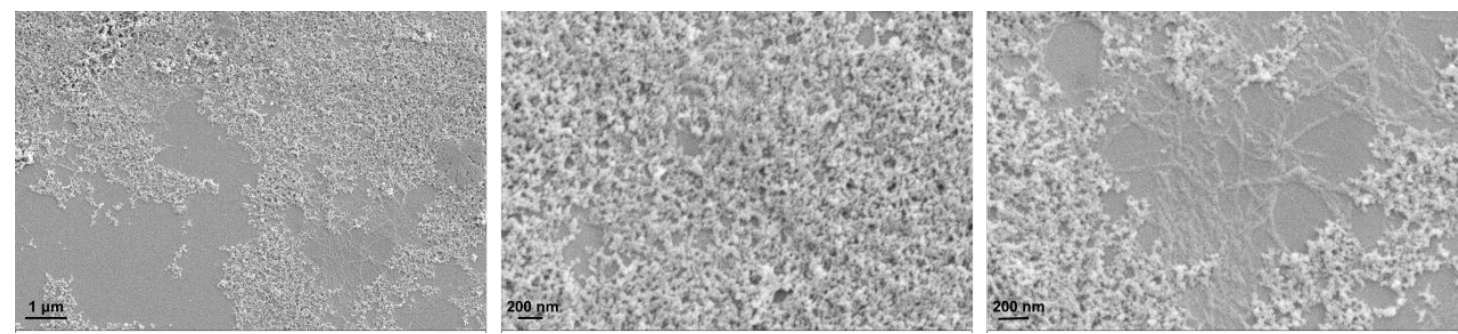

Figure S18. FE-SEM images of DC-CNFene-4 prepared with low ginger fiber $/ \mathrm{H}_{2} \mathrm{SO}_{4}$ ratio $(0.2 \mathrm{~g} / 80 \mathrm{~mL})$.
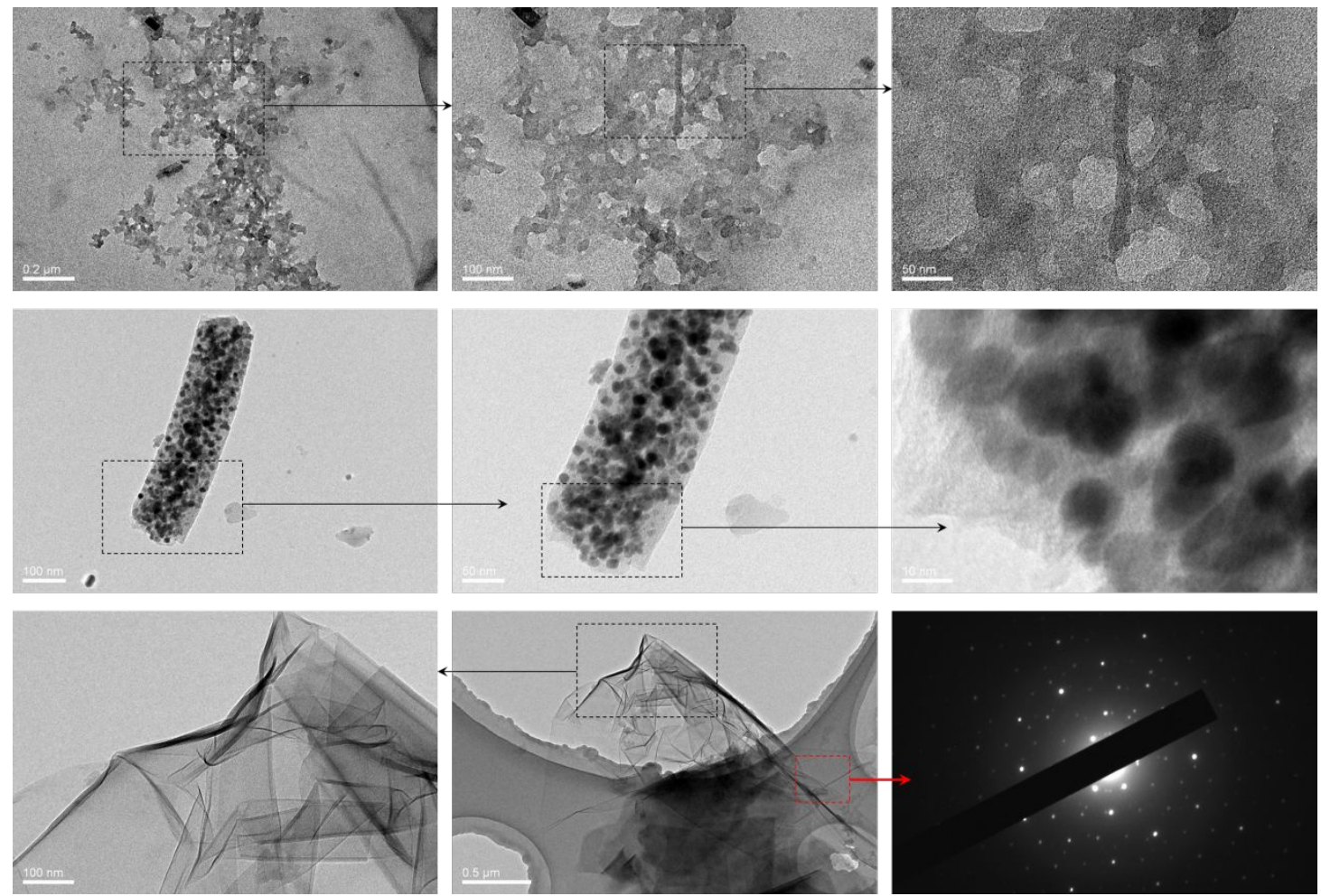

Figure S19. TEM images of DC-CNFene-4 prepared with low ginger fiber $/ \mathrm{H}_{2} \mathrm{SO}_{4}$ ratio $(0.2 \mathrm{~g} / 80 \mathrm{~mL})$ and the derived single layer graphene. 

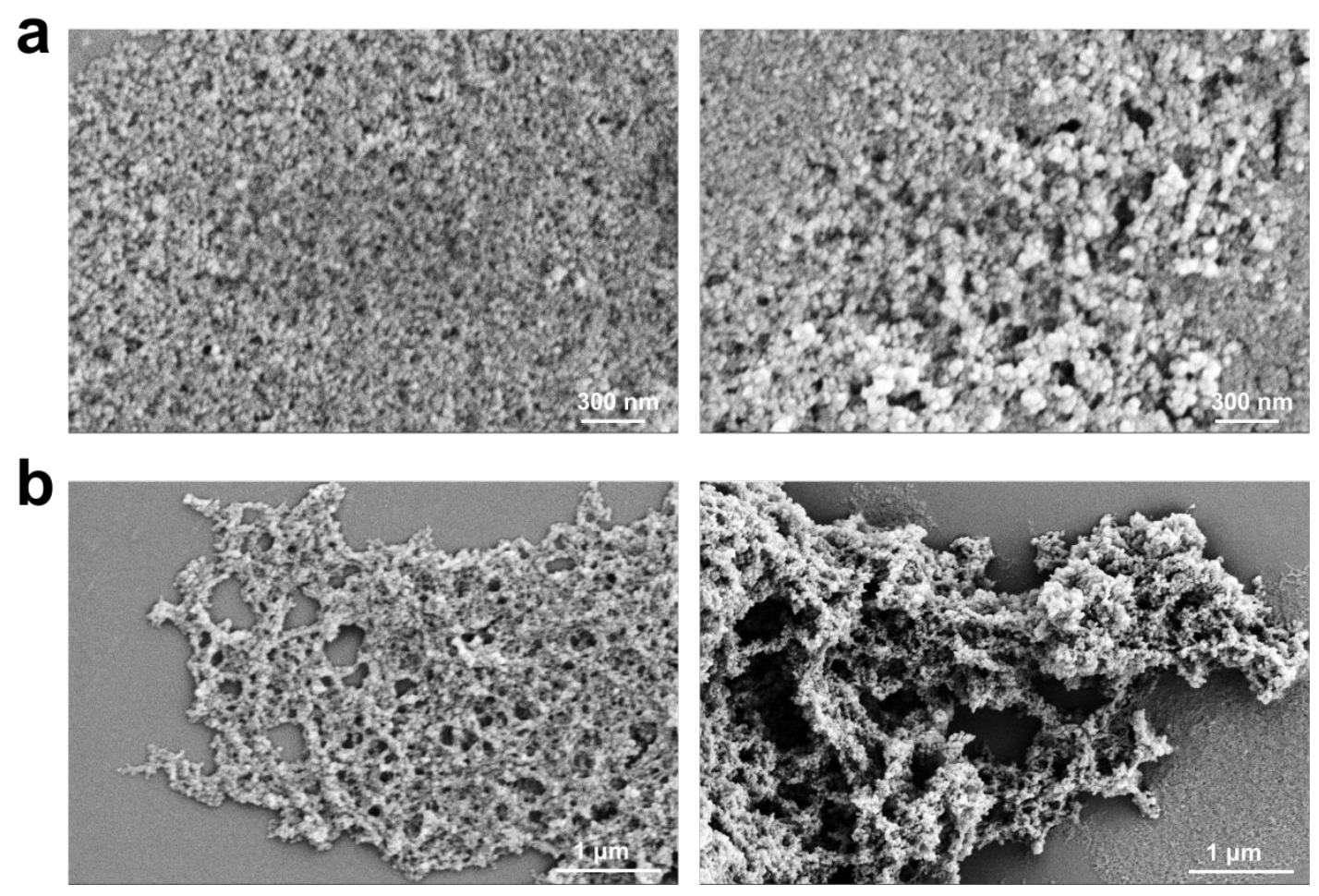

Figure S20. FE-SEM images of (a) DC-CNFene-Straw and (b) DC-CNFene-Bamboo. 

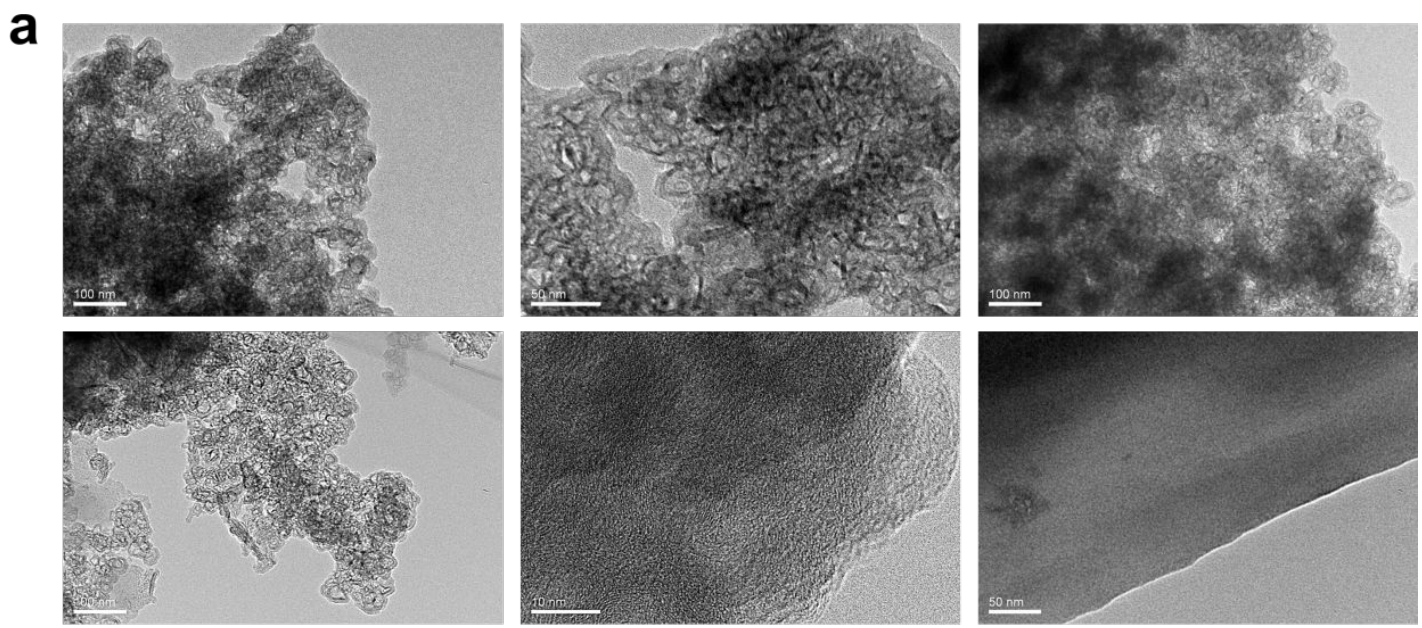

b
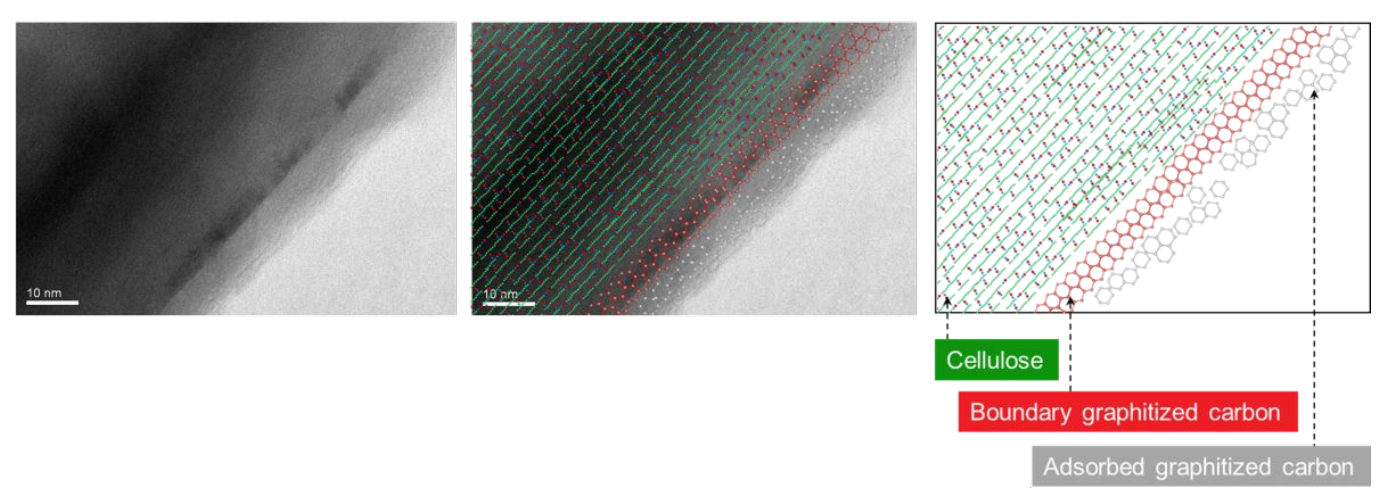

Figure S21. (a) TEM images of DC-CNFene-Straw and the derived graphene films.

(b) HRTEM images and schematic diagram of the DC-CNFene-Straw graphite carbon boundary. 

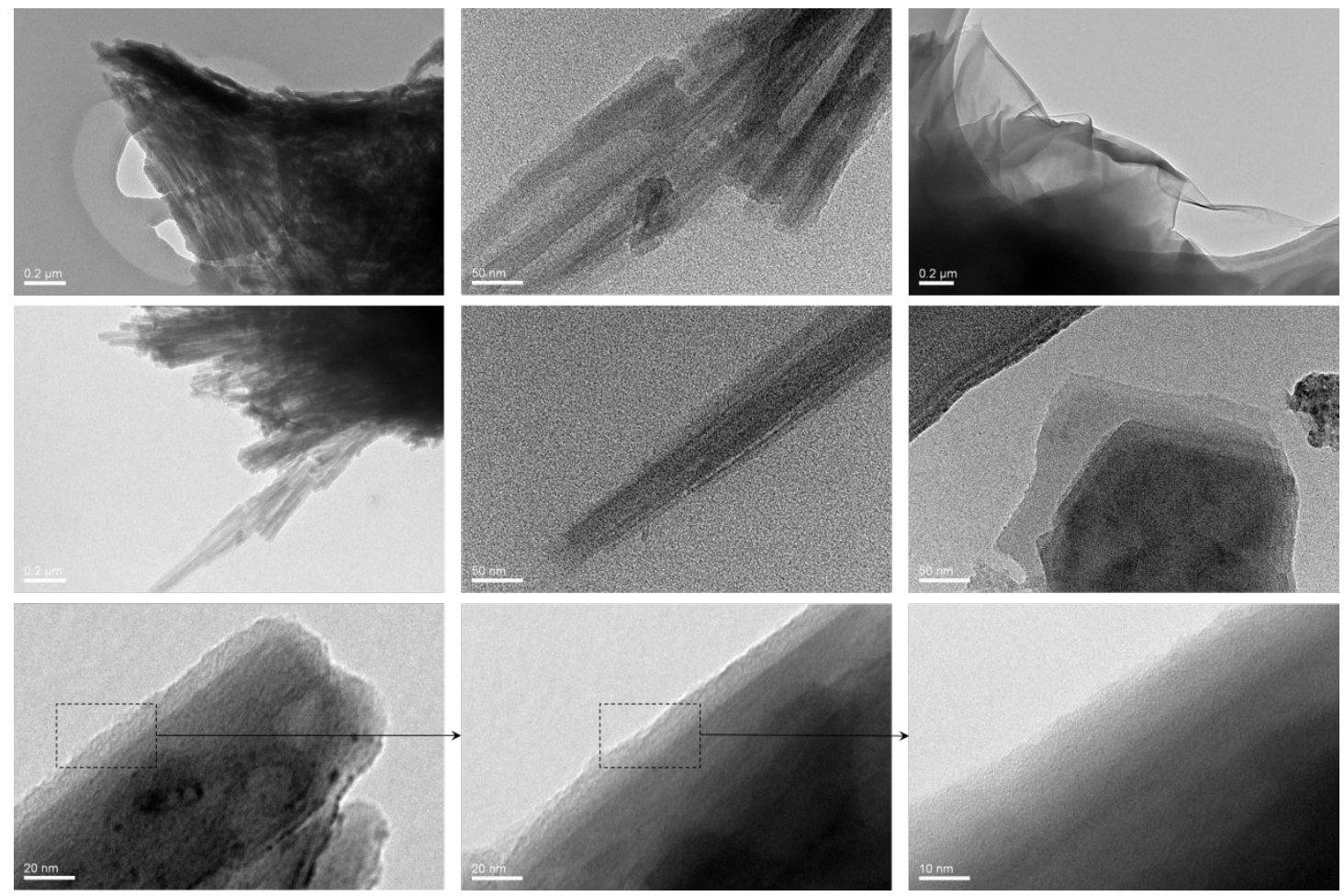

Figure S22. TEM images of DC-CNFene-Bamboo and the derived graphene films.
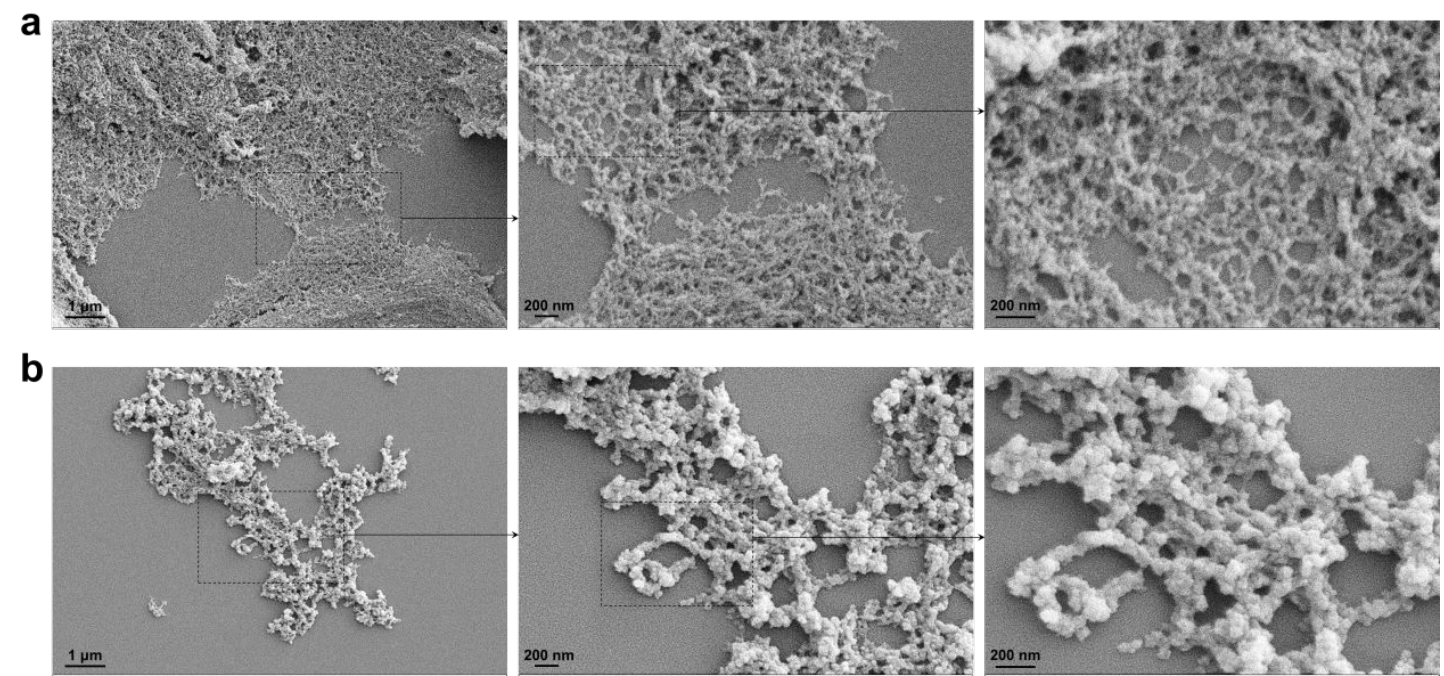

Figure S23. FE-SEM images of (a) DC-CNFene-Flax and (b) DC-CNFene-Wood. 

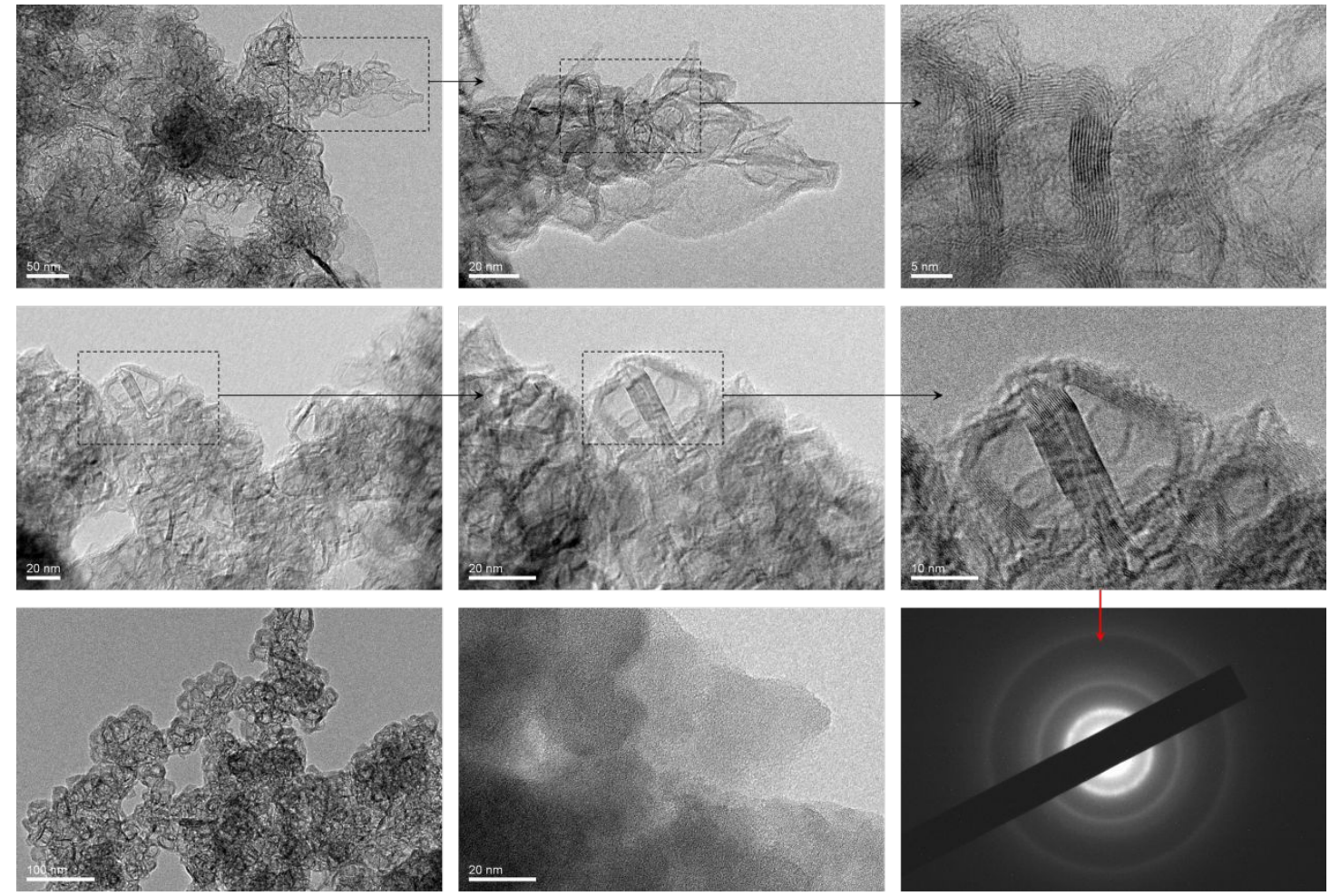

Figure S24. HRTEM images of DC-CNFene-Wood and the derived graphene films.
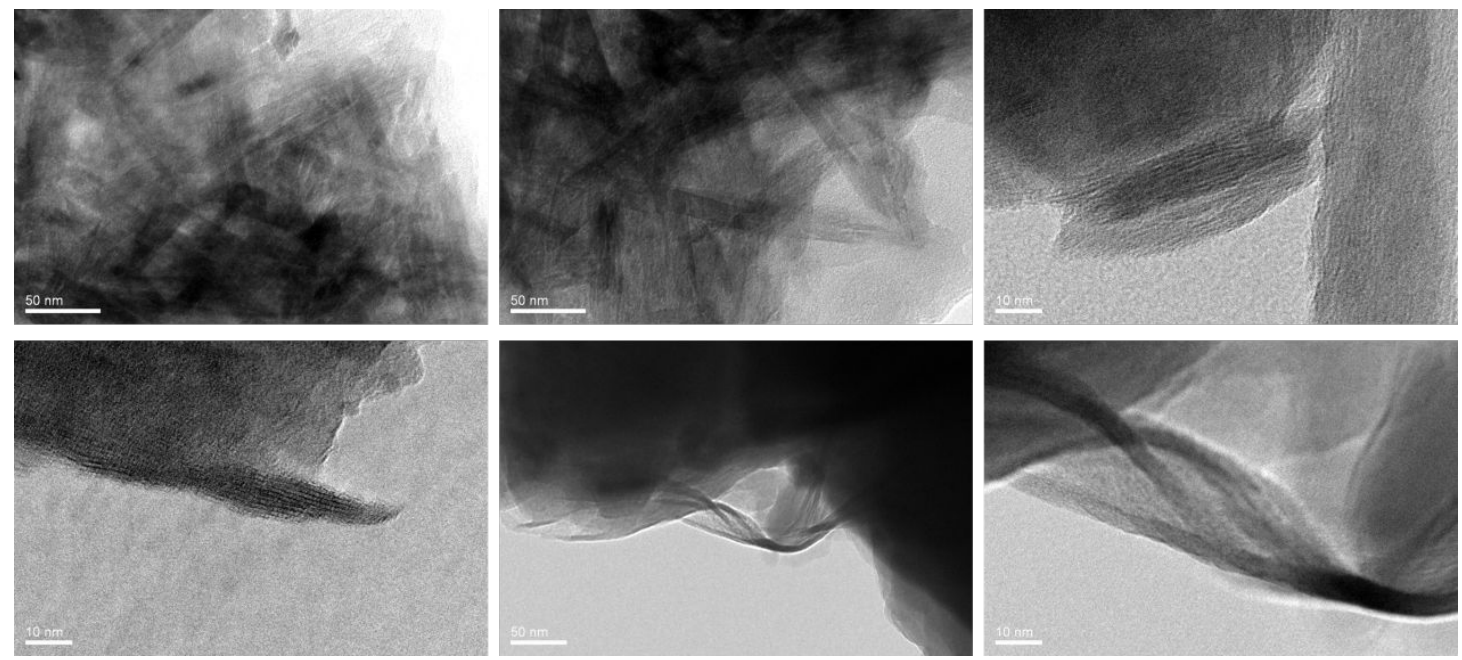

Figure S25. HRTEM images of DC-CNFene-Flax and the derived graphene films. 

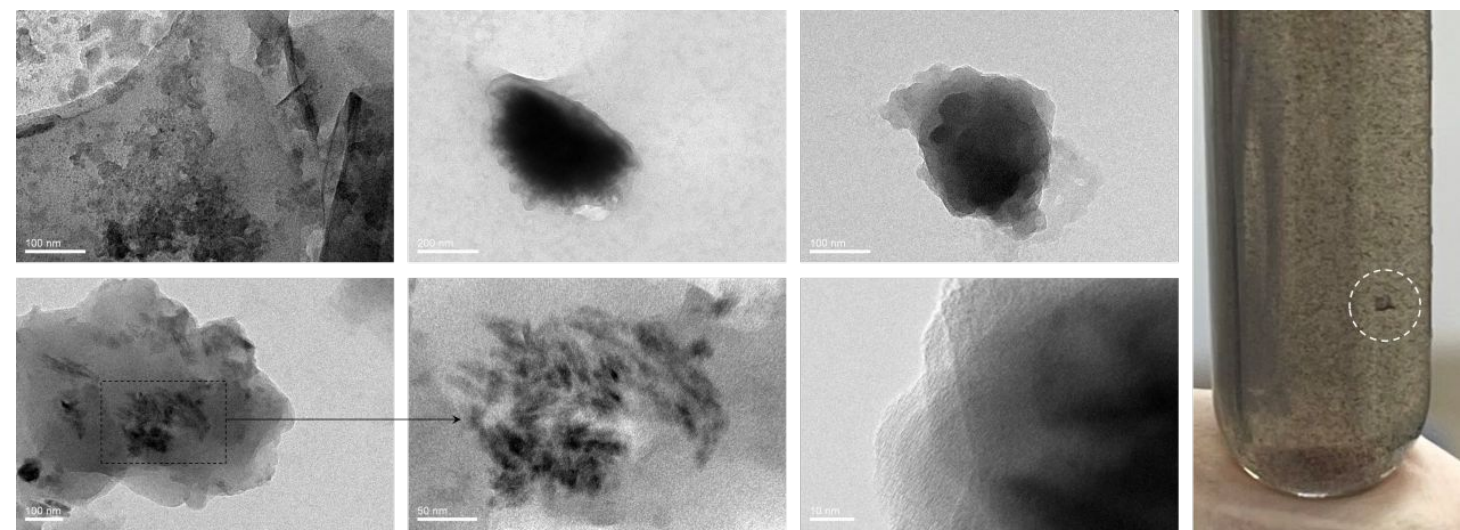

Figure S26. HRTEM images of lignin after four hours confined DC and a photo of a small amount of graphene film in the suspension after ultrasound.

Table S2. Dimensional statistics and yield of CNFenes.

\begin{tabular}{ccccc}
\hline Sample & $\begin{array}{c}\text { Length } \\
(\mathrm{nm})\end{array}$ & $\begin{array}{c}\text { Diameter } \\
(\mathrm{nm})\end{array}$ & $\begin{array}{c}\text { Graphitized carbon } \\
\text { layer thickness } \\
(\mathrm{nm})\end{array}$ & $\begin{array}{c}\text { Yield } \\
(\%)\end{array}$ \\
\hline DC-CNFene-1 & $247.31-641.69$ & $22.22-59.50$ & $\sim 19.28$ & $\sim 38.82$ \\
DC-CNFene-4 & $162.26-423.28$ & $18.50-31.35$ & $\sim 13.82$ & $\sim 33.41$ \\
DC-CNFene-Straw & N/A* & & $\sim 2.62$ & $\sim 25.36$ \\
DC-CNFene & 293.43-1293.03 & $23.65-52.80$ & $\sim 6.79$ & $\sim 35.22$ \\
- Bamboo & & & & $\sim 7.62$ \\
DC-CNFene-Flax & $81.12-271.67$ & $14.74-30.17$ & & $\sim 29.63$ \\
DC-CNFene-Wood & N/A* & $2.48-6.65$ & $\sim 2.01$ & $\sim 28.66$ \\
\hline
\end{tabular}

- More than 100 fibers per sample were counted.

* Difficult to distinguish the beginning and end of the sample, please refer to the TEM images. 

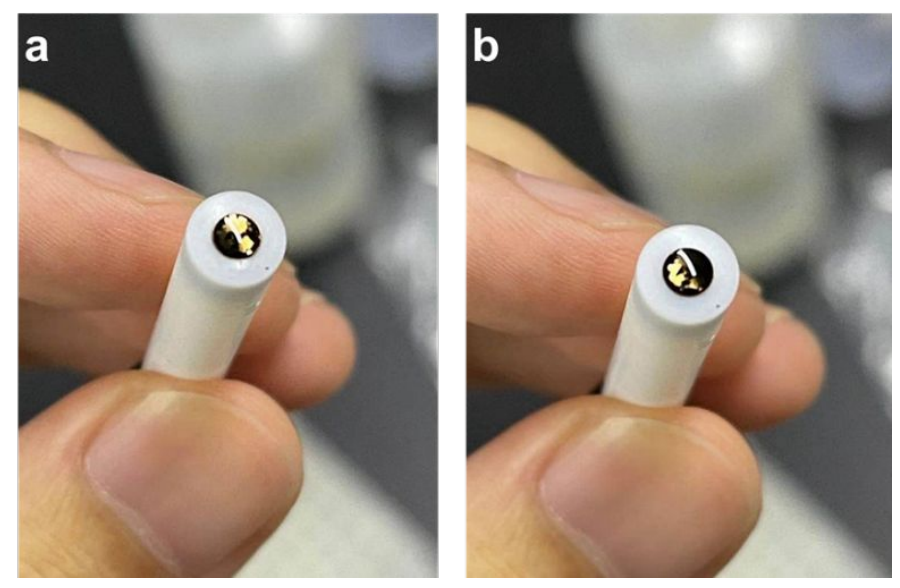

Figure S27. Photos of DC-CNFene-4 on the head of the glassy carbon electrode.
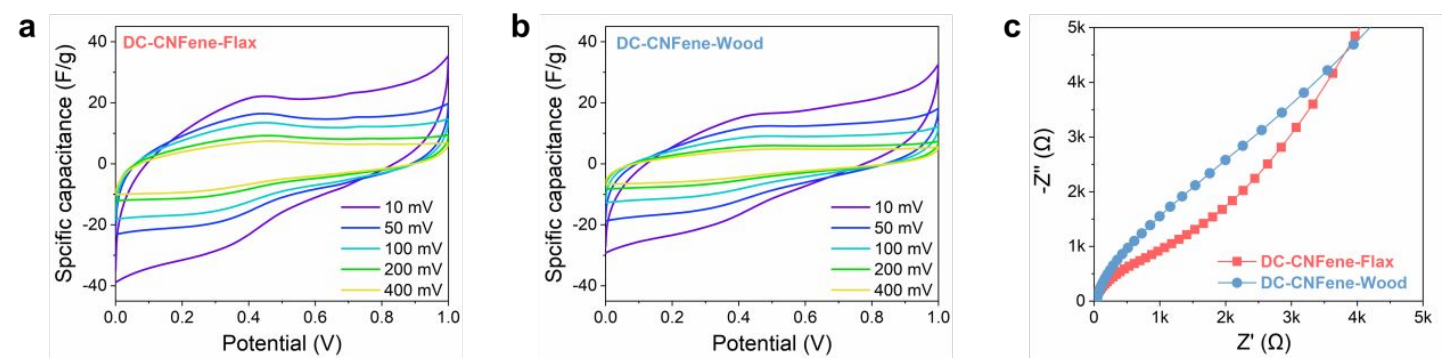

Figure S28. CV curves of (a) DC-CNFene-Flax and (b) DC-CNFene-Wood. (c)

Nyquist plots of DC-CNFene-Flax and DC-CNFene-Wood. 

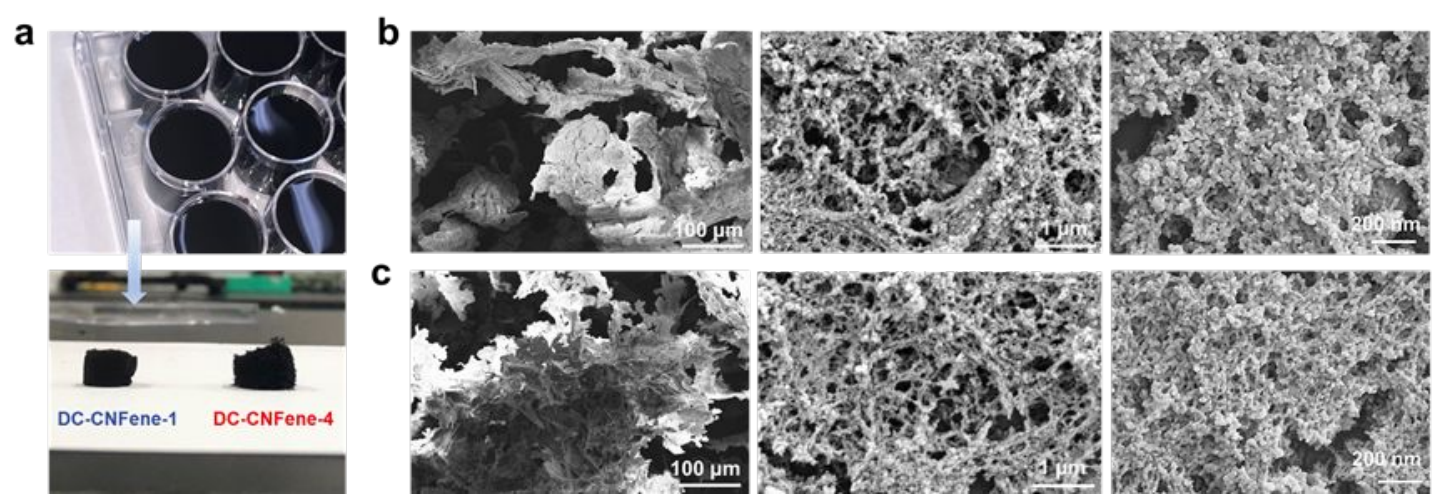

Figure S29. (a) CNFene suspensions and their freeze-dried aerogels, FE-SEM images of (b) DC-CNFene-1 and (c) DC-CNFene-4 aerogels.

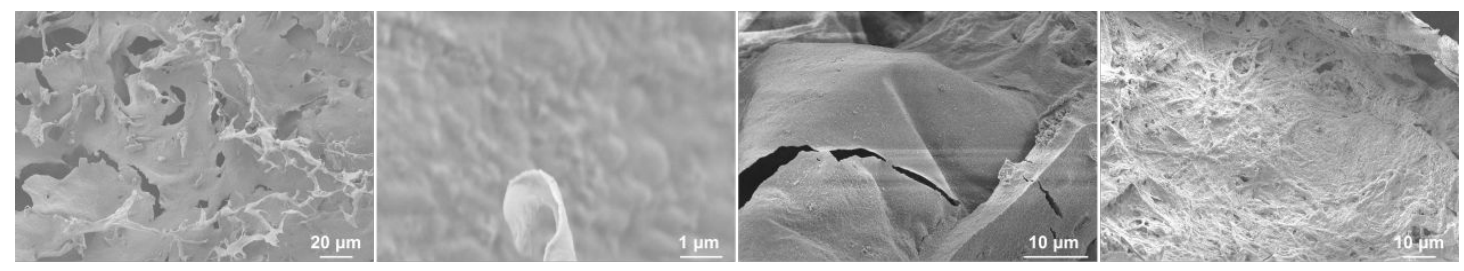

Figure S30. Graphene films in DC-CNFene-4 aerogels.
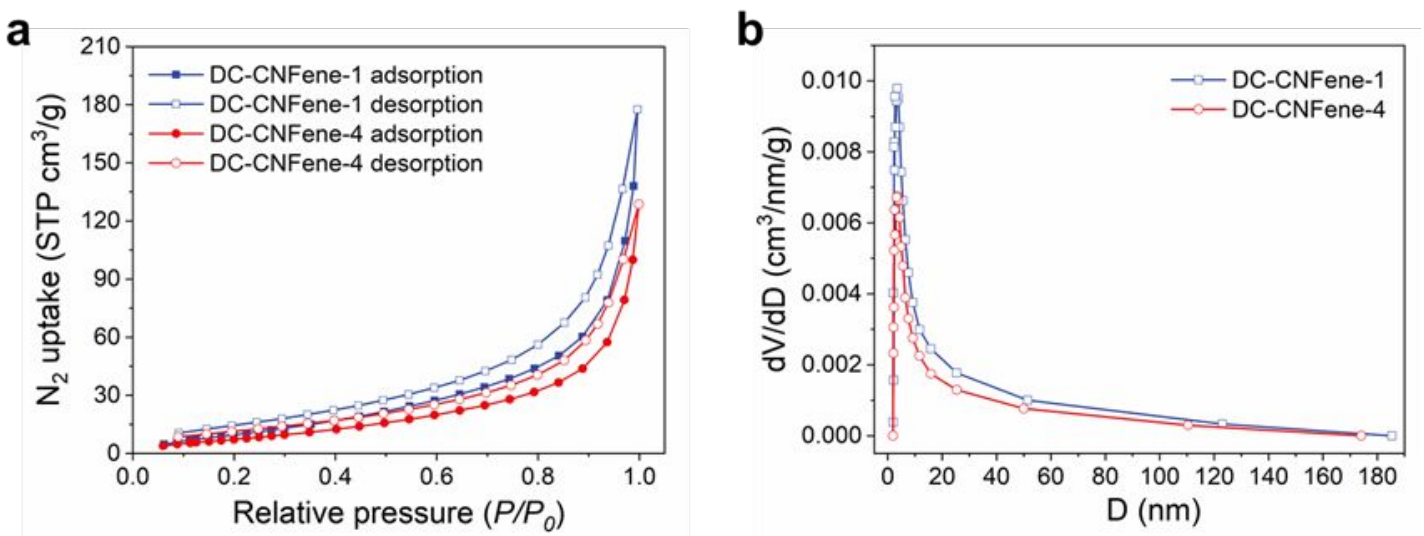

Figure S31. (a) $\mathrm{N}_{2}$ adsorption isotherms and (b) pore size distribution of DC-CNFene-1 and DC-CNFene- 4 . 


\section{References}

(1) Alemdar, A.; Sain, M., Biocomposites from wheat straw nanofibers: Morphology, thermal and mechanical properties. Composites Science and Technology 2008, 68 (2), $557-565$.

(2) Rovio, S.; Simolin, H.; Koljonen, K.; Sirén, H., Determination of monosaccharide composition in plant fiber materials by capillary zone electrophoresis. Journal of Chromatography A 2008, 1185 (1), 139-144.

(3) Moudood, A.; Rahman, A.; Öchsner, A.; Islam, M.; Francucci, G., Flax fiber and its composites: An overview of water and moisture absorption impact on their performance. Journal of Reinforced Plastics and Composites 2019, 38 (7), 323-339.

(4) Loudon, R., Theory of the resonance Raman effect in crystals. Journal de Physique 1965, $26(11), 677-683$.

(5) Morhange, J.; Beserman, R.; Balkanski, M., Raman study of the vibrational properties of implanted silicon. Physica Status Solidi (a) 1974, 23 (2), 383-391.

(6) Takahashi, J. I.; Makino, T., Raman scattering measurement of silicon-on-insulator substrates formed by high-dose oxygen-ion implantation. Journal of Applied Physics 1988, $63(1), 87-91$.

(7) De Wolf, I., Micro-Raman spectroscopy to study local mechanical stress in silicon integrated circuits. Semiconductor Science and Technology 1996, 11 (2), 139.

(8) Aspnes, D. E.; Studna, A., Dielectric functions and optical parameters of si, ge, gap, gaas, gasb, inp, inas, and insb from 1.5 to 6.0 ev. Physical Review B 1983, 27 (2), 985. (9) Liu, J.; Saw, R. E.; Kiang, Y.-H., Calculation of effective penetration depth in X- 
ray diffraction for pharmaceutical solids. Journal of Pharmaceutical Sciences 2010, 99 (9), 3807-3814.

(10) Kaky, K. M.; Şakar, E.; Akbaba, U.; Kasapoğlu, A. E.; Sayyed, M.; Gür, E.; Baki, S.; Mahdi, M., X-ray photoelectron spectroscopy (XPS) and gamma-ray shielding investigation of boro-silicate glasses contained alkali/alkaline modifier. Results in Physics 2019, 14, 102438. 